\title{
Reconstructing Aliased Frequency Spectra by Using Multiple Sample Rates
}

\author{
Maikel Huiskamp, Student Member, IEEE, Mark S. Oude Alink, Senior Member, IEEE, \\ Bram Nauta, Fellow, IEEE, Anne-Johan Annema, Member, IEEE and Harijot Singh Bindra, Member, IEEE
}

\begin{abstract}
A novel algorithm is presented that can resolve frequency ambiguity that arises from sampling a set of signals spanning more than a single Nyquist zone. The method uses two samplers, each sampling the same input signal with different (non-integer multiple) sampling rates. The algorithm is able to resolve frequency ambiguity and reconstruct signals with an orthogonal frequency basis spanning multiple Nyquist zones, provided that the aggregate information-bearing bandwidth of the signals is less than half the cumulative data converter sampling rates. This manuscript describes the theoretical background for the algorithm and validates it through measurements performed on a test-board comprising of two 10 bit analog-todigital converters clocked at two different (non-integer multiple) sample rates. Measurements show that even in the presence of aliasing, an orthogonal signal spanning multiple Nyquist zones can be fully reconstructed.
\end{abstract}

Index Terms-Analog-to-Digital conversion, aliasing, DFT, sampling, band-limited signals, Nyquist rate, time-interleaved ADC, sampling, reconstruction.

\section{InTRODUCTION AND PRIOR ART}

W ITH the advancements in technology the energy consumption for digital signal processing (DSP) has seen a tremendous decrease. Before physical (analog) signals can be processed by DSPs they have to be converted to the digital domain by an analog-to-digital converter (ADC). This digitization process involves the conversion of a continuous-time continuous-amplitude analog signal to a discrete-time discreteamplitude digital signal. The conversion from continuous-time to discrete-time may give rise to aliasing. Aliasing might cause different signal frequencies to become indistinguishable of each other, thus resulting in frequency ambiguity. Antialiasing filters circumvent aliasing by filtering frequencies outside the desired Nyquist zone (usually the first Nyquist zone is desired). However if these other (mostly higher) frequencies contain useful information, this information also gets removed.

The work in [1] solves frequency ambiguity due to aliasing by sub-sampling a sine wave with a time difference between two sampling clocks of the same frequency, resulting in a frequency-dependent phase shift. However, this solves the frequency ambiguity for a single tone only. The work in [2] proposes an approach that requires $(\mathrm{N}+1)$ co-prime timedelays and $(\mathrm{N}+1)$ ADCs for estimating $\mathrm{N}$ frequency components. But even in narrow band systems $\mathrm{N}$ is typically large, making this approach impractical.

Another method to estimate frequency content outside the desired Nyquist band is sub-sampling the input signal with different co-prime sample rates [3] and then calculating multiple DFTs corresponding to these sample rates. The frequency corresponding to each DFT bin can be related to the (corresponding) co-prime sample rates through a modulo operation and a residue. Using the Chinese remainder theorem [3] on this set of residues allows the determination of the unknown input frequencies. However, this method requires at least as many co-prime samplers as the number of frequencies to be estimated, which significantly increases system complexity.

The work in [4] demonstrates spectral estimation and identification of sinusoidal signals using co-prime samplers. In contrast to [3] the signal is sampled at sub-Nyquist rate by only two co-prime samplers to obtain two sparsely sampled data sets of the input signal. Spectral identification of the input signal is then performed by estimating the (time) average autocorrelation over multiple "snapshots" (time domain blocks) of the two sparsely sampled data sets. The accuracy of the spectral estimation (and auto-correlation) depends on the length of the time interval over which the averaging takes place. This results in a large latency in the overall signal estimation as highlighted in [4], thereby making it impractical to be used for real-time reconstruction of signals.

This paper proposes a deterministic algorithm to remove frequency ambiguity due to aliasing by sampling the signal at only two distinct non-integer multiples of each other sample rates. This allows the algorithm to unalias signals that may be spread over multiple Nyquist zones while simultaneously keeping the aggregated bandwidth close to the NyquistShannon sampling limit. The proposed algorithm requires signals that have an orthogonal frequency basis. This allows the signal to be sampled without any spectral leakage, which ensures that every spectral contribution occupies a single distinct frequency after sampling. The proposed algorithm could find applications in areas such as RF waveform characterization [5] (Appendix C) and built-in self-test of transceiver signal chains to improve digital pre-distortion and reliability, IQ reconstruction and other application scenarios as long as the frequencies of interest are known and the signal is orthogonal.

The structure of the paper is as follows. Section II revisits the constraints on the sample rate for baseband and bandlimited signals and shows the effect of frequency ambiguity due to aliasing. Section III introduces the proposed deterministic algorithm for resolving multiple simultaneous bandlimited signals. Section IV compares the proposed algorithm with other techniques. Section V discusses the limitations of this algorithm under non-ideal conditions. Section VI shows experimental verification of the algorithm, along with a qualitative comparison with conventional sampling techniques. Although all examples and test signals used in this paper are 
orthogonal frequency-division multiplexing (OFDM) signals , the proposed algorithm can be used to detect and reconstruct signals with any orthogonal frequency basis as shown in section III. Section VII summarizes the findings in this paper.

\section{FREQUENCY AMBIGUITY DUE TO ALIASING}

Frequency ambiguity arises when two aliases or the baseband signal and an alias fold on top of each other. When sampling a signal aliasing can occur, which can result in signals folding on top of each other making them indistinguishable, hence resulting in frequency ambiguity. This work assumes that $x(t)$ contains uniformly spaced orthogonal discrete-frequency sub-carriers with frequency spacing $f_{o}$. Most modern communication systems use signals with some form of orthogonal frequency basis for modulation such as OFDM and orthogonal on-off keying (OOOK). This work aims at more efficiently approaching the Nyquist-Shannon limit for input signals with an orthogonal frequency basis that are spread over multiple frequency bands (e.g. multi-band OFDM). To retain orthogonality of the sub-carriers in each symbol, both the symbol period must be a multiple of $\frac{1}{f_{o}}$ and the complex value of the sub-carriers must remain constant within a symbol period [6].

$x(t)$ can be described as a set of $L$ orthogonal sub-carriers, which may be non-contiguous in the frequency domain. Since $x(t)$ is a band-limited signal it can also be represented by

$$
x(t)=\sum_{l=1}^{L}\left(A_{l} e^{j 2 \pi S_{l} f_{o} t}+A_{l}^{*} e^{-j 2 \pi S_{l} f_{o} t}\right)
$$

where $0 \leq t<\frac{1}{f_{o}}, S$ is the set of $L$ down-converted modulated sub-carriers, $S_{l} \in \mathbb{N}$ is the $l^{\text {th }}$ element in $S, A$ is a set containing the complex values (magnitude and phase) of the sub-carriers in $S, A_{l}$ is the $l^{\text {th }}$ element in $A,|S|$ and $|A|$ are the ranks of $S$ and $A$ respectively and $L=|S|=|A|$. Please note that in (1) $A_{l}$ can be a complex number. Different orthogonal signals (such as OOOK or MFSK) differ in the M-ary encoding scheme, which corresponds to a different complex value $A_{l}$ in (1) e.g. $A_{l}=\{0,1\}$ in OOOK. The proposed algorithm does not depend on the chosen modulation scheme, as long as the modulation scheme has an orthogonal frequency basis.

The straightforward way to convert an analog signal to a time-discrete signal without any aliasing is to sample the signal at a frequency higher than twice the highest frequency component in the signal, such that

$$
f_{s} \geq 2 f_{o} \cdot \max (S)
$$

However this is inefficient as the frequency content between the $N$ band-limited signals lacks any information, yet is processed.

According to the Nyquist-Shannon theorem [7], an analog signal can be reconstructed without loss of information if $f_{s}$ is higher than twice the bandwidth of the signal. The minimum sampling rate $f_{\mathrm{s}, \min }$ for $x(t)$ is therefore:

$$
f_{\mathrm{s}, \min }>2|S| f_{o} .
$$

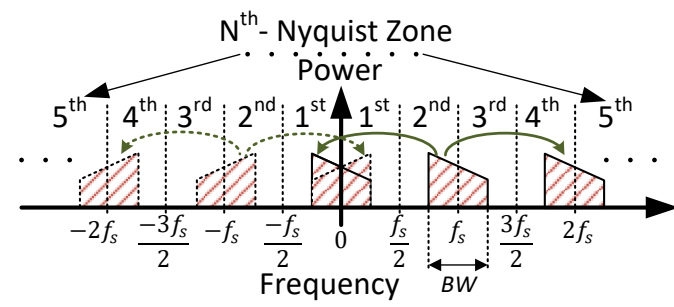

Fig. 1: Aliasing of a band-limited signal centred around $f_{s}$ to the baseband for the case e.g. $r=\{-1,1\}$ in (5).

If $f_{s}$ is chosen to satisfy the Nyquist-Shannon limit as defined in (3), then aliasing can occur [8], [9], since $2|S| f_{o} \leq$ $f_{s} \leq 2 f_{o} \cdot \max (S)$.

Sampling $x(t)$ uniformly at a rate $f_{s}$ results in:

$$
y(t)=x(t) \cdot p(t),
$$

where $p(t)$ is a series of Dirac delta pulses:

$$
p(t)=\sum_{r=-\infty}^{\infty} \delta\left(t-\frac{r}{f_{s}}\right)
$$

where $r \in \mathbb{Z}$. The Fourier transform of $y(t)$ can be written as:

$$
Y(f)=f_{s} \sum_{r=-\infty}^{\infty} X\left(f-r f_{s}\right)
$$

where $X(f)$ is the Fourier transform of $x(t)$.

The work in [8] and [10] showed that there are upper and lower bounds on $f_{s}$ when directly sampling multiple bandlimited signals that are spread over multiple Nyquist zones. If these boundary conditions for $f_{s}$ are not satisfied, then some frequency components alias on top of each other making them indistinguishable, resulting in frequency ambiguity as illustrated in Fig. 1.

IQ reconstruction can remove a specific frequency ambiguity due to aliasing [8], as shown in Fig. 2. However, frequency components in higher Nyquist zones (frequency band from $\frac{(N-1) f_{s}}{2}$ to $\frac{N f_{s}}{2}$ where $N>1$, see Fig. 2), all fold into the first Nyquist zone. As shown in Fig. 2(e) these signals (from multiple Nyquist zones) become indistinguishable and cannot be recovered with IQ reconstruction. We present an algorithm that allows more flexibility in allocation of component carriers which can be spread over multiple Nyquist zones.

\section{Multi-Rate UnAliasing}

This section presents a mathematical description of an algorithm that can resolve frequency ambiguity due to aliasing, by using multiple sample rates that are non-integer multiples of one another. We denote this algorithm as the multi-rate unaliasing (MRU) algorithm.

\section{A. MRU Algorithm}

In this section we present an algorithm that utilizes two samplers with distinct sampling frequencies to enable demodulation of orthogonal signals from several Nyquist zones. Simultaneously it allows an aggregated sample rate close to 

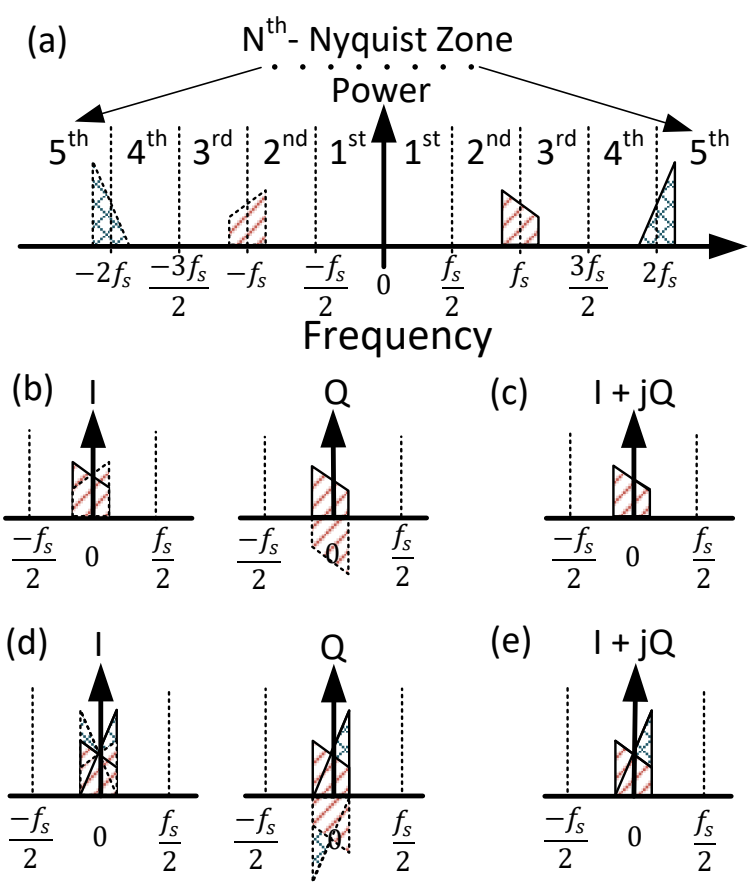

(c)

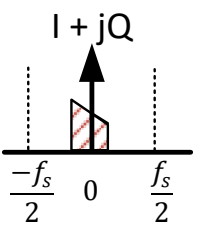

(e)

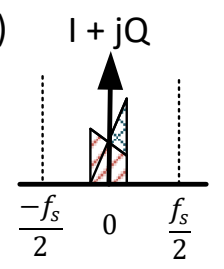

Fig. 2: (a) Two bandpass signals in different Nyquist zones where the frequency components of the band-limited signals are on either side of the sampling frequency $f_{s}$. (b) I and Q power spectra after sampling when only the signal in (a) centred around $f_{s}$ is present. (c) $\mathrm{I}+\mathrm{jQ}$ spectrum from (b) where the original input spectra can be recovered (d) I and Q power spectra after sampling when both signals in (a) are present (e) $\mathrm{I}+\mathrm{jQ}$ spectrum from (d) where the original input spectra cannot be distinguished from each other

the aggregated bandwidth of $2 f_{o}|S|$. Sampling the signal at different sample rates creates unique aliasing patterns for the same input signal spectrum as described by (5). A system of independent linear equations can be formulated that describes these aliasing patterns, allowing for reconstruction of the input spectrum. The limitations of the MRU algorithm on the maximum aggregated bandwidth and maximum input signal frequency are discussed.

The discrete-time sequence $d[n]$ at the output of the sampler contains the weight of the impulse train in the uniformly sampled signal $y(t)$ in equation (4) for sampling intervals $T_{s}$ :

$$
d[n]=y\left(n T_{s}\right)
$$

where $T_{s}=\frac{1}{f_{s}}$ and $n$ is the sample index. When sampled at $f_{s}=M f_{o}$ the duration of a symbol period equal to $\frac{1}{f_{o}}$, $d[n]$ has length $M$, which will be used as the DFT length. We assume that these M-samples belong to a single symbol to retain orthogonality between symbols. This ensures that the spectral content of each sampled symbol is mapped uniquely to a single DFT bin, allowing reconstruction by the MRU algorithm. The $M$-point DFT of $d[n]$ is:

$$
D[k]=\frac{1}{M} \sum_{n=0}^{M-1} d[n] e^{-j 2 \pi \frac{n}{M} k} \quad 0 \leq k \leq M-1 .
$$

By combining (6), (7) and using

$$
\frac{1}{M} \sum_{n=0}^{M-1} e^{ \pm j 2 \pi S_{l} \frac{n}{M}} e^{-j 2 \pi k \frac{n}{M}}= \begin{cases}1 & \text { when } k= \pm S_{l} \mp r M \\ 0 & \text { otherwise. }\end{cases}
$$

$D[k]$ can be written as

$$
\begin{array}{r}
D[k]=\sum_{l=1}^{L}\left(\sum _ { r = - \infty } ^ { \infty } \left(A_{l} \delta\left(S_{l}-k-r M\right)+\right.\right. \\
\left.\left.A_{l}^{*} \delta\left(S_{l}+k-r M\right)\right)\right) .
\end{array}
$$

$D[k]$ is the discrete-frequency representation of the input signal $x(t)$ and its aliases after sampling at a rate $f_{s}=M f_{o}$. We now reformulate (9) in vector form as:

$$
D[k]=\mathbf{P}[k] \mathbf{A}
$$

where $\mathbf{A}$ is a $2 L \times 1$ column-vector containing both $A_{l}$ and $A_{l}^{*}$ and where $\mathbf{P}[k]$ is a $1 \times 2 L$ row-vector describing the contributions of $\mathbf{A}$ to the value of $D[k]$, and contains information about the aliasing pattern. $\mathbf{P}[k]$ is ordered in such a way that the even and odd elements respectively describe the contributions of $\mathbf{A}$ and $\mathbf{A}^{*}$. This representation is chosen for convenience and does not further affect the algorithm. Thus:

$$
\begin{gathered}
P[k, 2 l]=\sum_{r=-\infty}^{\infty} \delta\left(S_{l}-k-r M\right), \\
P[k, 2 l+1]=\sum_{r=-\infty}^{\infty} \delta\left(S_{l}+k-r M\right)
\end{gathered}
$$

where $0 \leq k \leq M-1$ and $P[k, l] \in\{0,1\}$.

Combining the $M$ linear equations for $D[k]$ into one matrix yields

$$
\mathbf{D}=\mathbf{P A}
$$

where $\mathbf{P}$ is an $M \times 2 L$ matrix. The aliasing pattern of $\mathbf{A}$ to $\mathbf{D}$ depends on the signal frequencies relative to the sampling frequency. Changing the sampling frequency changes the aliasing pattern, resulting in a different $\mathbf{P}$.

Fig. 3 shows the conceptual diagram of the MRU algorithm. The switches $S_{1}$ and $S_{2}$ are samplers that are clocked respectively at two different (non-integer multiple) sample rates $M_{1} f_{o}$ and $M_{2} f_{o}$.To demonstrate the effectiveness of MRU, the input signal $x(t)$ is directly generated from a signal source and is sampled by both $\mathrm{S} 1$ and $\mathrm{S} 2$. In a practical system $x(t)$ would be the signal after frequency translation. This results in two complex DFTs $\mathbf{D}_{\mathbf{M}_{1}}$ and $\mathbf{D}_{\mathbf{M}_{2}}$ after performing the DFT on $d_{M_{1}}[n]$ and $d_{M_{2}}[n] . \mathbf{D}_{\mathbf{M}_{1}}$ and $\mathbf{D}_{\mathbf{M}_{2}}$ are column vectors with sizes $M_{1} \times 1$ and $M_{2} \times 1$ respectively. The two DFTs are concatenated into a column vector $\mathbf{D}_{\mathbf{c}}$ of length $\left(M_{1}+M_{2}\right) \times 1$ resulting in

$$
\mathbf{D}_{\mathbf{c}}=\left[\begin{array}{l}
\mathbf{D}_{\mathrm{M}_{1}} \\
\mathbf{D}_{\mathrm{M}_{2}}
\end{array}\right]
$$

In mathematical terms concatenation is equivalent to the collection of output DFTs from both the samplers and vertically appending the DFTs to create a new matrix.

The two distinct sets of aliasing patterns corresponding to the sample frequencies $M_{1} f_{o}$ and $M_{2} f_{o}\left(\mathbf{P}_{\mathbf{M}_{\mathbf{1}}}\right.$ and $\left.\mathbf{P}_{\mathbf{M}_{\mathbf{2}}}\right)$ are formed by using (11) and (12) and are concatenated as

$$
\mathbf{P}_{\mathrm{c}}=\left[\begin{array}{l}
\mathbf{P}_{\mathrm{M}_{1}} \\
\mathbf{P}_{\mathrm{M}_{2}}
\end{array}\right]
$$




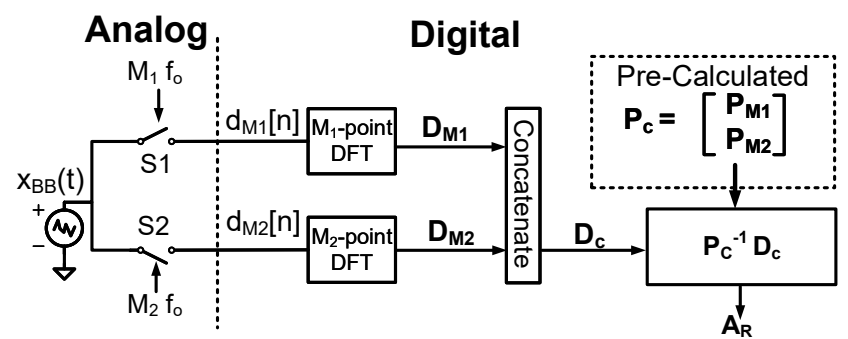

Fig. 3: Schematic overview of MRU. The two samplers $S_{1}$ and $S_{2}$ are clocked at $M_{1} f_{o}$ and $M_{2} f_{o}$ respectively. After a period of $\frac{1}{f_{o}}, M_{1}$ and $M_{2}$ point DFTs are calculated and combined into $\mathbf{D}_{\mathbf{c}}$. The vector $\mathbf{D}_{\mathbf{c}}$ and matrix $\mathbf{P}_{\mathbf{c}}$ allow for reconstructing the complex value (magnitude and phase) vector $\mathbf{A}$

where $\mathbf{P}_{\mathbf{c}}$ has size $\left(M_{1}+M_{2}\right) \times 2 L$. The reconstructed complex value vector $\mathbf{A}_{\mathbf{R}}$ can then be described as

$$
\mathbf{A}_{\mathbf{R}}=\mathbf{P}_{\mathbf{c}}^{-1} \mathbf{D}_{\mathbf{c}}
$$

where $\mathbf{P}_{\mathbf{c}}^{-\mathbf{1}}$ is the left inverse of $\mathbf{P}_{\mathbf{c}} . \mathbf{A}_{\mathbf{R}}$ contains the estimated complex value (magnitude and phase) information of the sub-carriers in the signal $x(t)$. Performing an IFFT on the sub-carriers corresponding to the signal of interest in $\mathbf{A}_{\mathbf{R}}$ will result in the respective time domain estimate of $x(t)$. An example demonstrating MRU is shown in Appendix A.

The maximum bandwidth that can be reconstructed depends on $\operatorname{rank}\left(\mathbf{D}_{\mathbf{c}}\right)$. The $\operatorname{rank}\left(\mathbf{D}_{\mathbf{c}}\right)$ equals the number of unique samples taken by the two samplers over the sampling interval $T_{s}$. The total number of samples equals $\left(M_{1}+M_{2}\right)$, whereas the common (coinciding) number of samples equals $\operatorname{gcd}\left(M_{1}, M_{2}\right)$, where $\operatorname{gcd}(\cdot)$ represents the greatest common divisor. Then the $\operatorname{rank}\left(\mathbf{D}_{\mathbf{c}}\right)=\left(M_{1}+M_{2}\right)-\operatorname{gcd}\left(M_{1}, M_{2}\right)$, which allows to resolve $\left\lfloor\frac{M_{1}+M_{2}-\operatorname{gcd}\left(M_{1}, M_{2}\right)}{2}\right\rfloor$ frequency components. For orthogonal frequency components and a sampling interval $T_{s}=\frac{1}{f_{o}}$ the bandwidth that can be properly converted equals

$$
B W_{\max }=\left(\left\lfloor\frac{M_{1}+M_{2}-\operatorname{gcd}\left(M_{1}, M_{2}\right)}{2}\right\rfloor-1\right) \cdot f_{o}
$$

Note that in contrast to co-prime sampling systems [4], $\operatorname{gcd}\left(M_{1}, M_{2}\right)=1$ is not required in our sampling system. The only requirement is that $M_{1}$ and $M_{2}$ are non-integer multiples of each other, as otherwise (17) reduces to:

$$
B W_{\max }=\left\lfloor\frac{\max \left(M_{1}, M_{2}\right)}{2}\right\rfloor \cdot f_{o} \text { for } \frac{M_{1}}{M_{2}} \text { or } \frac{M_{2}}{M_{1}} \in \mathbb{N}
$$

which is in accordance to the well known Nyquist theorem.

In a conventional Nyquist sampling system a single continuous signal band with bandwidth $B W$ is sampled by a single sample frequency $f_{s}$. MRU allows for the bandwidth (17) to be spread over the frequency range for which the aliasing pattern $\mathbf{P}$ is uniquely defined. This frequency range is:

$$
0 \leq f \leq \operatorname{lcm}\left(M_{1}, M_{2}\right) \cdot f_{o}
$$

where $\operatorname{lcm}(\cdot)$ represents the least common multiple.

The aliasing patterns as described by (11) and (12) have a periodicity of $M$. For the two sample rates $M_{1}$ and $M_{2}$ this results in an overall periodicity of the combined aliasing pattern that is the $1 \mathrm{~cm}$ of the two sample rates. This means that the input signal frequencies can be spread over the frequency range given by (19) before MRU cannot solve frequency ambiguity due to aliasing.

One of the considerations in the selection of $M_{1}$ and $M_{2}$ is the total aggregated bandwidth, see (17), which is maximized when $\operatorname{gcd}\left(M_{1}, M_{2}\right)=1$ (i.e. $M_{1}$ and $M_{2}$ are co-prime). Another point of consideration is the maximum frequency span over which the aggregated bandwidth can be spread out, see (19), which again is maximized when $M_{1}$ and $M_{2}$ are coprime. Lastly, it follows that for co-prime $M_{1}$ and $M_{2}$ the aggregated sample rate $\left(M_{1}+M_{2}\right) \cdot f_{o}$ can remain close to the Nyquist-Shannon limit of $2 f_{o}|S|$ as long as all sub-carriers contribute to an increase of $\operatorname{rank}\left(\mathbf{P}_{\mathbf{c}}^{-\mathbf{1}}\right)$ in (16).

\section{QUALITATIVE COMPARISON OF MRU WITH OTHER TECHNIQUES}

\section{A. Time-interleaving versus MRU}

Time-interleaving of $N$ sub-rate ADCs increases the aggregated bandwidth [11]. This requires a sample clock with $\mathrm{N}$ equally spaced phases, where the phase accuracy requirement increases $\mathrm{N}$-fold to maintain the distortion performance [12], [13]. The increase in aggregated bandwidth increase the frequency span of a Nyquist zone, however the problem of aliasing will still occur (but at a higher frequency). In contrast to time-interleaving MRU allows an increase in aggregated bandwidth by using multiple sample rates that are non-integer multiples of one another. Any timing mismatch between the sample clocks can be fully compensated for by the MRU algorithm as described in section V-B1. However MRU needs the two samplers to both have a bandwidth equal or higher than the full signal bandwidth, where a time interleaved ADC only requires a single high-bandwidth sampler. The sub-ADCs in a time-interleaved ADC can have a lower bandwidth and thus consume less power.

MRU also allows for flexibility in the allocation of the aggregated bandwidth to different Nyquist zones. It is possible to combine MRU together with time-interleaving, however each time-interleaved $\mathrm{ADC}$ has to be calibrated as in a conventional time-interleaved system before MRU can be applied.

\section{B. Anti-aliasing versus $M R U$}

Increasing the order of the anti-aliasing filter or increasing the oversampling ratio increases the amount of suppression for signals outside the first Nyquist zone. However, any aliasing that occurs will still degrade the signal to noise ratio (SNR) of the reconstructed signal. MRU is not used to suppress the signals outside the first Nyquist zone and can be combined with multiple bandpass filters with distinct center frequencies. This will prevent strong signals outside the first Nyquist zone from saturating the ADC so that MRU can be used to unalias them. If after filtering a strong alias (such as an out-of-band interferer) folds into the band of interest, the wanted signal and the alias become indistinguishable. If the strong alias does not saturate the ADC, MRU can resolve this frequency ambiguity as long as the original frequency band of the alias is known, increasing the SNR of the wanted signal. MRU can be expanded to include more than two samplers, increasing 
the aggregated bandwidth over which the aliasing pattern $\mathbf{P}$ is uniquely defined (19). Using more samplers with non-integer sample rates would therefore allow unaliasing of multiple interferers from the wanted signal which would otherwise be impossible with just conventional filtering [14], [15].

The programmability in [14] does allow multiple Nyquist zones to be covered, however the power consumption of such a programmable anti-alias filter is orders of magnitude more than the two ADCs used in our MRU algorithm. Even with the analog front-end driver in medium resolution state-of-the-art ADCs [16], the power consumption is lower than the stateof-the-art anti-aliasing filter for comparable analog bandwidth and SNR[15]. Furthermore, the filters in [14], [15] require a clock signal that has orders of magnitude higher frequency than the bandwidth of the signal itself. In comparison to this the presented MRU algorithm is based on sub-sampling and does not require a high frequency clock signal. However, MRU requires an additional sub-sampling clock frequency and extra digital signal processing.

\section{Potential advantages of $M R U$}

MRU allows for flexibility in the allocation of frequency bands, thereby providing more programmability compared to solutions with a single sampler. In a conventional sampling system aliasing can be prevented by changing the sampling frequency. However, in case of a minimum sampling rate, the precision of the sample rate must increase for signals in higher Nyquist zones to prevent aliasing [8]. When multiple bandpass signals are present, the range of sample frequencies that do not aliase will also decrease [9]. In contrast to conventional systems the clock rates in MRU have to be multiples of $f_{o}$, meet the aggregated bandwidth requirement in (17) and the bandpass signals should be in the frequency range as defined in (19) to prevent any aliasing that cannot be resolved.

Other potential advantages of MRU are the reduced samplerate (and thus the digital data rate), since the aggregated sample rate can be significantly lower than $2 f_{o}(\max (S)-\min (S))$. Furthermore MRU might in some scenarios replace the antialiasing filter as long as the occupied frequencies before aliasing are known and the signal does not clip the ADC. Furthermore, the proposed MRU algorithm requires samples over one single symbol period $\frac{1}{f_{o}}$, just as a conventional sampling scheme. The latency in [4] is approximately $K$ times longer compared to conventional sampling, where $K$ is the number of snapshots (300 and 2000 in [4] ) used in averaging.

The MRU algorithm can also be extended to work directly with IQ signals. These systems typically already use two ADCs, hence the extra ADC overhead is removed when applying MRU in these systems. The modified equations for the reconstruction of IQ signals are given in Appendix B, along with the simulation results.

\section{IMPACT OF NON-IDEALITIES}

So far, it was assumed that the two samplers are ideal. However, in an actual implementation the digitized output suffers from non-idealities, e.g. gain mismatch, time delays, offsets, quantization and thermal noise. Any deviation from the ideal conditions degrades performance. This section discusses the impact of the most important non-ideal effects, using the reconstruction of OFDM signals as demonstration vehicle.

\section{A. Inaccuracy propagation: the matrix condition number}

The quantization noise and thermal noise of the ADC causes errors in the computation of $\mathbf{D}_{\mathbf{c}}$. These errors propagate through the system of linear equations resulting in an error in the reconstructed complex value (magnitude and phase) vector, $\mathbf{A}_{\mathbf{R}}$. The sensitivity of $\mathbf{A}_{\mathbf{R}}$ to these (small) errors depends on the condition number of $\mathbf{P}_{\mathbf{c}}$, as $\mathbf{P}_{\mathbf{c}}$ has to be inverted. The condition number of a matrix is defined (in $\mathrm{dB}$ ) as:

$$
\kappa=20 \log \left(\frac{\sigma_{\max }}{\sigma_{\min }}\right) \geq 0 \mathrm{~dB}
$$

where $\sigma_{\max }$ and $\sigma_{\min }$ are the largest and smallest singular values of the matrix.

A higher value of $\kappa$ indicates a higher propagation of estimation errors in $\mathbf{D}_{\mathbf{c}}$ to the elements in $\mathbf{A}_{\mathbf{R}}$ and thus indicates a higher in error vector magnitude (EVM). Fig. 4 shows the impact of $\kappa$ on the SNR of the reconstructed frequency spectra assuming two samplers with respective sample rates of $4.86 \mathrm{MHz}\left(M_{1}=324\right)$ and $5.13 \mathrm{MHz}\left(M_{2}=342\right)$ which yields an aggregated bandwidth of $\mathrm{BW}_{\max }=4.86$ $\mathrm{MHz}$. For the simulation results in this figure, the aggregated signal bandwidth is increased from $490 \mathrm{kHz}$ to $\mathrm{BW}_{\max }$. For $B W<\frac{\max \left(M_{1}, M_{2}\right) f_{o}}{2}$ the signal conversion can be done by a single sampler, resulting in $\kappa=0$. For $B W \geq \frac{\max \left(M_{1}, M_{2}\right) f_{o}}{2}$ the MRU algorithm is used resulting in $\kappa>0$.

Fig. 4 shows both the condition number of $\mathbf{P}_{\mathbf{c}}$ and the maximum SNR degradation for different aggregated signal bandwidths for these sample rates. Additive white gaussian noise is used as the signal $x(t)$ to determine the SNR degradation due to the condition number. The SNR degradation reported is the difference between the noise power of an ideal sampling system (switches $S_{1}$ and $S_{2}$ ) operating at twice the aggregated bandwidth and that of the MRU algorithm. In order to show the effect of the condition number of the matrix $\mathbf{P}_{\mathbf{c}}$ on the SNR, the aggregated bandwidth of the MRU algorithm is varied. The ADCs sample rates are fixed at $4.86 \mathrm{MS} / \mathrm{s}$ and $5.13 \mathrm{MS} / \mathrm{s}$. The ADCs in MRU might have a higher sample frequency than in a Nyquist rate sampling system, resulting in a lower noise power per DFT bin in MRU. This effect has been compensated for in the simulation results by normalizing the simulated SNR by the oversampling ratio. The maximum difference in the noise power between the additive white gaussian noise and the MRU output is reported in Fig. 4. Figure 4 illustrates that a higher condition number $\kappa$ thus results in a higher SNR degradation, thus reducing the accuracy of the reconstruction. Note that in general the SNR degradation depends on the relative aggregated bandwidth with respect to the aggregated sample rate, hence a wide-band system can achieve similar results.

\section{B. ADC Non-idealities and Calibration}

This section discusses the most typical non-idealities for AD converters to be present. With ideal sampling, the algorithm 


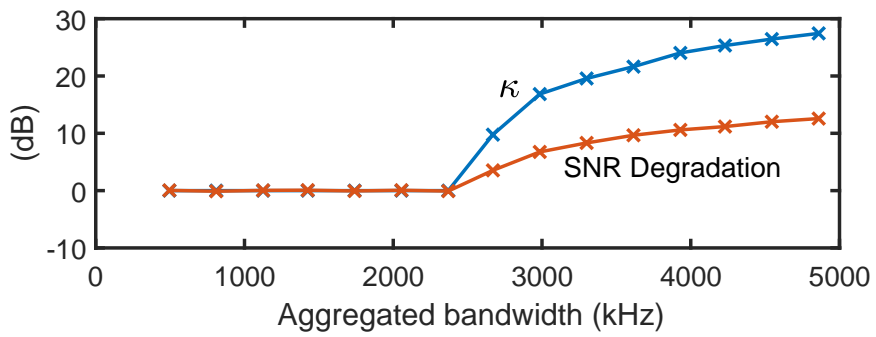

Fig. 4: Condition number of $\mathbf{P}_{\mathbf{c}}$ and maximum SNR degradation for different aggregated signal bandwidths. The ADC sample rates are $4.86 \mathrm{MHz}$ and 5.13 $\mathrm{MHz}$. The SNR gain due to oversampling is compensated for in these results.

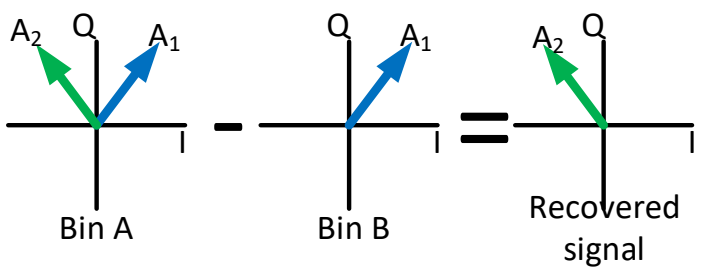

Fig. 5: DFT Bin A contains both the complex vectors $A_{1}$ and $A_{2}$ and DFT bin $\mathrm{B}$ only contains $A_{1}$. By subtracting Bin $\mathrm{B}$ from Bin A the complex vector $A_{2}$ is recovered for ideal sampling.

performs the operation as shown in Fig. $5 ; A_{2}$ is calculated by subtracting Bin B from Bin A resulting exactly in the actual vector $A_{2}$.

1) Timing Mismatch: In Section III it is assumed that the two different sampling frequencies $M_{1} f_{o}$ and $M_{2} f_{o}$ have a common rising edge at the beginning of the sample sequence, resulting in $d_{M_{1}}[1]=d_{M_{2}}[1]$. This ensures that the complex values $\mathbf{A}$ in $\mathbf{D}_{\mathbf{M}_{1}}$ and $\mathbf{D}_{\mathbf{M}_{2}}$ retain the same phase.

In case there is no common rising edge at the beginning of the sample sequence, and therefore no common first sample, $d_{M_{1}}[n]$ and $d_{M_{2}}[n]$ are effectively sampled with a constant time delay $\Delta t$ between them.

Another source of timing mismatch is the path delay difference between individual paths. The switches $S_{1}$ and $S_{2}$ shown in Fig. 3 are part of a linear time variant system, therefore path delay mismatch between the two samplers $S_{1}$ and $S_{2}$ can be treated in the same manner as the static clock timing mismatch.

Time delay results in a phase rotation of $D[k]$ and is described by:

$$
\begin{array}{r}
D_{\Delta t}[k]=\sum_{l=1}^{L}\left(\sum _ { r = - \infty } ^ { \infty } \left(A_{\varphi, l} \delta\left(S_{l}-k-r M\right)+\right.\right. \\
\left.\left.A_{\varphi, l}^{*} \delta\left(S_{l}+k-r M\right)\right)\right)
\end{array}
$$

where $A_{\varphi, l}=e^{j \varphi_{o} S_{l}} A_{l}, A_{\varphi, l}^{*}=e^{-j \varphi_{o} S_{l}} A_{l}^{*}$ and $\varphi_{o}=$ $2 \pi f_{o} \Delta t$. Fig. 7 illustrates the impact of a time mismatch.

The resulting DFT vector from (21) is described by:

$$
\mathbf{D}_{\Delta t}=\mathbf{P}_{\varphi} \mathbf{A}
$$

where $\mathbf{P}_{\varphi}$ is described by:

$$
P_{\varphi}[k, 2 l]=\sum_{r=-\infty}^{\infty}\left(e^{j \varphi_{0} S_{l}} \delta\left(S_{l}-k-r M\right)\right)
$$

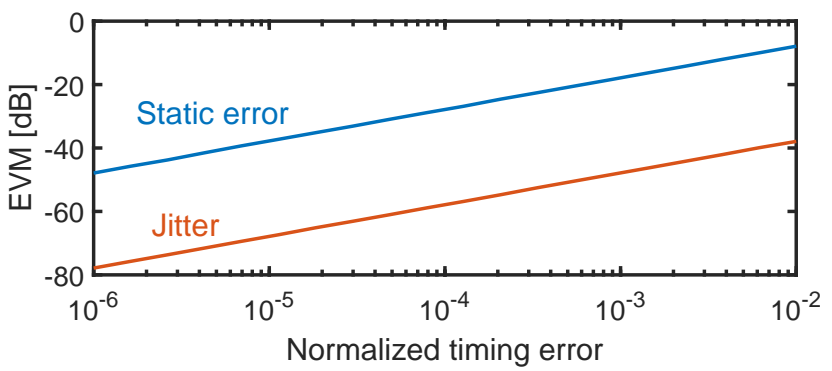

Fig. 6: Simulated EVM versus normalized timing error for a static timing error and jitter. The static time delay error is defined between the common rising edges of the two sample clocks and the error is normalized to $\frac{1}{f_{o}}$, which. The timing error caused by jitter is added to both the ADC sampling clocks. The standard deviation of the jitter is normalized to the ADC sampling period.

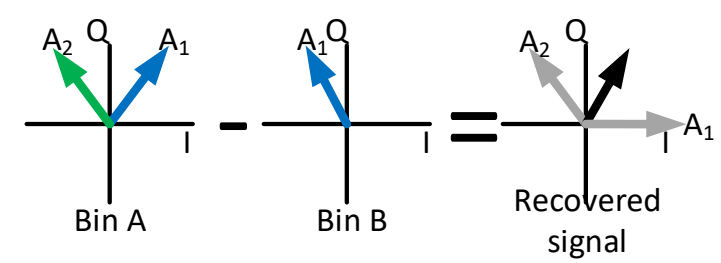

Fig. 7: Signal recovery in the presence of time difference between the sample points at the start of the beat pattern. Here DFT Bin A contains both the complex vectors $A_{1}$ and $A_{2}$ and DFT bin $\mathrm{B}$ only contains a time shifted version of $A_{1}$. By subtracting Bin $\mathrm{B}$ from Bin A the complex vector $A_{1}$ is only partially cancelled and still contributes to the estimation of $A_{2}$.

$$
P_{\varphi}[k, 2 l+1]=\sum_{r=-\infty}^{\infty}\left(e^{-j \varphi_{0} S_{l}} \delta\left(S_{l}+k-r M\right)\right) .
$$

The calibration for timing mismatch is done by providing a known single sinusoidal signal as the input and comparing the phase of the two output DFTs at the input sinusoidal frequency. The timing mismatch is calculated from the phase difference by

$$
\Delta t=\frac{\Delta \phi}{2 \pi f_{\text {sin }}}
$$

where $\Delta \phi$ is the phase difference between the two output DFTs at the input sinusoidal frequency, $f_{\sin }$. This is a onetime calibration.

Fig. 6 shows the (MATLAB) simulated effect of static timing mismatch between the first rising edges of both sample clocks on the expected EVM when using MRU. Here the timing mismatch is normalized to the symbol period $\frac{1}{f_{o}}$. The EVM degradation without any calibration due to timing mismatch is $10 \mathrm{~dB} /$ decade, which behaves similarly to SNR degradation due to timing mismatch in non-calibrated twochannel time-interleaved ADCs [12].

Another source of timing mismatch is jitter in the ADC clocks, which causes dynamic errors in $\Delta t$. In conventional ADCs jitter decreases the SNR of the sampled waveform, hence it will also decrease the accuracy of the MRU algorithm. Conventionally jitter (due to noise) is a random process, which can often be described by a Gaussian distribution with zero mean and standard deviation $\sigma_{t}$. Fig. 6 shows the simulated EVM for different $\sigma_{t}$ normalized to the ADC sample period.

2) Gain error: The MRU algorithm uses two samplers, or two ADCs. These ADCs may have differences in their transfer from the analog input voltage to the digital code, resulting in 


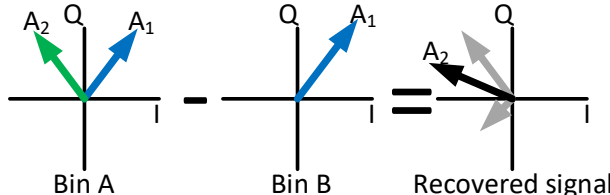

Fig. 8: Signal recovery in the presence of gain mismatch between the ADCs. DFT Bin A contains complex vectors $A_{1}$ and $A_{2}$ and DFT bin B contains a scaled version of $A_{1}$. By subtracting Bin $\mathrm{B}$ from Bin A the complex vector $A_{1}$ is only partially cancelled and still contributes to the estimation of $A_{2}$.

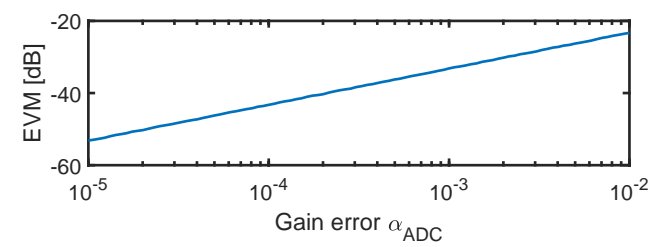

Fig. 9: Simulated EVM versus gain error $\alpha_{A D C}$. In this simulation only a single ADC has a gain error, and the other ADC has unity gain.

a static gain error. The MRU algorithm however assumes the contributions of $\mathbf{A}$ to $\mathbf{D}_{\mathbf{M}_{1}}$ and $\mathbf{D}_{\mathbf{M}_{2}}$ to be equal, leading to errors, see Fig. 8. In this work the ADCs are assumed to have unity gain. To compensate the gain error $\alpha_{\mathrm{ADC}}, \mathbf{D}_{C}$ must be normalized to the gain of the $\operatorname{ADC}\left(1+\alpha_{\mathrm{ADC}}\right)$ :

$$
\mathbf{D}_{C, \text { norm }}=\left[\begin{array}{c}
\frac{\mathbf{D}_{M 1}}{1+\alpha_{\mathrm{ADC} 1}} \\
\frac{\mathbf{D}_{M 2}}{1+\alpha_{\mathrm{ADC} 2}}
\end{array}\right] \text {. }
$$

A static gain error can be compensated for in the digital domain and is performed by comparing the magnitude of two output DFTs at the input sinusoidal frequency.

Fig. 9 shows the simulated effect of the gain error $\alpha_{A D C}$ on EVM when using MRU. In this simulation it is assumed that only a single ADC has a gain error, and the other ADC has unity gain. The EVM degradation due to the gain error $\alpha_{A D C}$ is $10 \mathrm{~dB} /$ decade, which is similar to SNR degradation due to gain errors in time-interleaved ADCs [12]. Furthermore, simulations demonstrate that dynamic gain errors follow the same trend as static gain errors.

3) Offset: Offset of both ADCs end up in the first bins of $\mathbf{D}_{\mathbf{M}_{1}}$ and $\mathbf{D}_{\mathbf{M}_{2}}$. The resulting dataset is obtained by concatenating the two DFT datasets and not by switching between the outputs of the two ADCs. Therefore there is no up-conversion of the DC-offset; consequently offset errors can be compensated in the same manner as for a traditional ADC. The offsets for the two ADCs are obtained from the DC bin of the respective output DFTs.

\section{Vi. MeAsurement Results}

Fig. 10 shows the measurement test board containing two energy and area-efficient 10 bit SAR ADCs as presented in [17], which are used in this demonstrator since they were available in this project. Note that the algorithm is not limited to low speed ADCs and can also be used with high speed ADCs. The two ADCs share the same differential inputs and are clocked separately. The ADC architecture is an asynchronous one wherein the falling edge of the sample clock denotes the

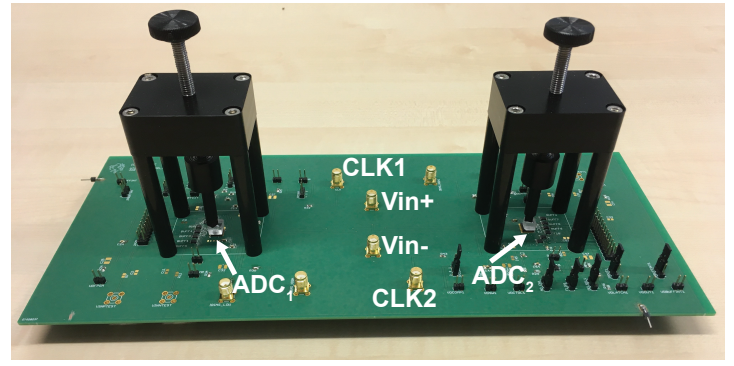

Fig. 10: Photograph of the measurement PCB.

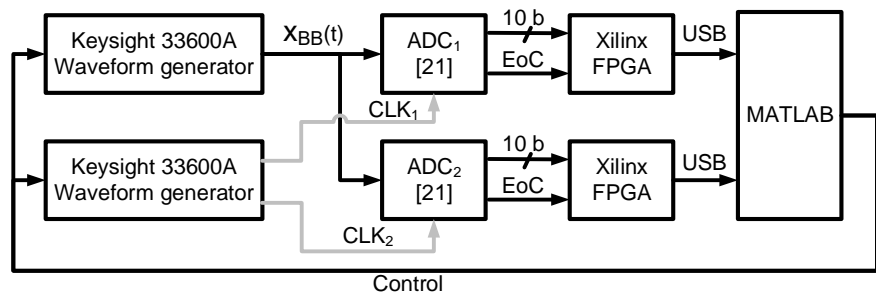

Fig. 11: Block diagram of the measurement setup.

end of the sampling period and the internal logic controller is self-timed and generates all the ADC conversion cycles.

The accuracy of the ADCs limit the maximum achievable EVM and the relation between SNR and EVM $_{\text {RMS }}$ is $\mathrm{EVM}_{\mathrm{RMS}} \approx \mathrm{SNR}^{-\frac{1}{2}}$ [18] for a noise limited system. For the ADCs used in the measurements, the signal to noise and distortion ratio (SINAD) $>56 \mathrm{~dB}$, hence the EVM can be determined up to an accuracy of approximately $0.16 \%$ which is sufficient for most applications. For example 256-QAM LTE requires an EVM $<3 \%$ [19] for which typically 8 to 12 bit ADCs are used [20], [21].

Fig. 11 shows the block diagram of the measurement setup. At the end of each conversion, the signal EoC indicates to the FPGA to latch the data for reconstruction. The ADC output bits are collected by the respective FPGAs (serial-toparallel conversion), which then connects to a computer with MATLAB running the MRU algorithm.

To demonstrate the effectiveness of MRU three different measurements are performed. For these measurements OFDM signals are used, since they are a well known type of orthogonal signal. Appendix $\mathrm{C}$ discusses the characterization of the harmonics of an RF-signal as another use case. The first measurement demonstrates the ability of MRU to separate an OFDM signal where due to sampling sub-carriers are aliased into the first Nyquist zone, hence becoming indistinguishable from each other. The second measurement demonstrates that MRU can be used to reconstruct aliased signals outside the first Nyquist zone, where a single OFDM band covers higher Nyquist zones. The third measurement combines the results of the two previous measurements for a dual-band signal, demonstrating the ability of MRU to simultaneously resolve for frequency ambiguity and individually reconstructs the band-pass signals. Although the test signals used in this section are OFDM, MRU in general can be used to detect and reconstructs signals with any orthogonal frequency basis. 
TABLE I: Aliasing pattern when the OFDM signal is sampled at $4.86 \mathrm{MS} / \mathrm{s}$ as described by (5). Only aliasing to the first Nyquist zone is shown ( $r=-1$ and $\mathrm{r}=0$ ).

\begin{tabular}{|c|c|c|}
\hline & Input frequency & Frequency after sampling \\
\hline \multirow{2}{*}{$r=-1, f+f_{s}$} & $-2.595 \mathrm{MHz}$ & $2.265 \mathrm{MHz}$ \\
\cline { 2 - 3 } & $-2.415 \mathrm{MHz}$ & $2.445 \mathrm{MHz}$ \\
\hline \multirow{2}{*}{$r=0, f$} & $2.415 \mathrm{MHz}$ & $2.415 \mathrm{MHz}$ \\
\cline { 2 - 3 } & $2.595 \mathrm{MHz}$ & $2.595 \mathrm{MHz}$ \\
\hline
\end{tabular}

TABLE II: Aliasing pattern when the OFDM signal is sampled at $5.13 \mathrm{MS} / \mathrm{s}$ as described by (5). Only aliasing to the first Nyquist zone is shown ( $\mathrm{r}=-1$ and $\mathrm{r}=0$ ).

\begin{tabular}{|c|c|c|}
\hline \multirow{2}{*}{$r=-1, f+f_{s}$} & Input frequency & Frequency after sampling \\
\cline { 2 - 3 } & $-2.595 \mathrm{MHz}$ & $2.535 \mathrm{MHz}$ \\
\hline \multirow{2}{*}{$r=0, f$} & $-2.415 \mathrm{MHz}$ & $2.715 \mathrm{MHz}$ \\
\cline { 2 - 3 } & $2.415 \mathrm{MHz}$ & $2.415 \mathrm{MHz}$ \\
\cline { 2 - 3 } & $2.595 \mathrm{MHz}$ & $2.595 \mathrm{MHz}$ \\
\hline
\end{tabular}

\section{A. Reconstruction of an aliased signal centred around $f_{s} / 2$}

To demonstrate the algorithm a $180 \mathrm{kHz}$ BW OFDM signal centred around $2.505 \mathrm{MHz}$ and ranging from $2.415 \mathrm{MHz}$ to $2.595 \mathrm{MHz}$ is used as a test signal. This signal mimics a continues signal down-mixed to baseband, having no out-ofband frequency components. The OFDM signal consists of 12 sub-carriers spaced at $15 \mathrm{kHz}$ modulated with a 16-QAM constellation and a Null-carrier at $2.505 \mathrm{MHz}$ for a total of 13 sub-carriers. The Null-carrier is used as a reference for reconstruction and does not influence the performance of the MRU algorithm. Since the sub-carrier spacing is $15 \mathrm{kHz}, f_{o}$ $=15 \mathrm{kHz}$ to retain orthogonality of the sub-carriers after sampling. The input signal spectrum is shown in Fig. 12(a).

This OFDM signal is simultaneously sampled by $A D C_{1}$ and $A D C_{2}$ at $4.86 \mathrm{MS} / \mathrm{s}\left(M_{1}=324\right)$ and $5.13 \mathrm{MS} / \mathrm{s}\left(M_{2}=342\right)$ respectively, since these sample rates are close to the maximum sample rates of the available ADCs. However, the MRU algorithm can be extended to (significantly) higher sample rates and correspondingly a higher aggregated input signal bandwidth. After sampling the input signal with these sample rates aliasing occurs. For simplicity only aliasing to the positive half of the first Nyquist zone is considered and is shown in Table I. The sampled signal frequency span ranges from 2.265 $\mathrm{MHz}$ to $2.595 \mathrm{MHz}$ and the frequency range from $2.415 \mathrm{MHz}$ to $2.445 \mathrm{MHz}$ now contains multiple aliased carriers to a single DFT bin. Similarly the aliasing for $A D C_{2}$ is calculated and shown in Table II. Here the frequencies from $2.535 \mathrm{MHz}$ to $2.595 \mathrm{MHz}$ contain multiple aliased carriers to a single DFT bin. Fig. 12(b) and 12(c) show the output spectra $\left|\mathbf{D}_{\mathbf{M}_{1}}\right|^{2}$ and $\left|\mathbf{D}_{\mathbf{M}_{2}}\right|^{2}$ of respectively $A D C_{1}$ and $A D C_{2}$ zoomed in around the signal of interest for a single OFDM symbol out of a 200 symbol sequence. After sampling the data is passed to the MRU algorithm to reconstruct the input signal. $M_{1}$ and $M_{2}$ are selected such that the two ADCs operate near to their maximum throughput rate. If the ADCs can operate at higher sample rates, $M_{1}$ and $M_{2}$ can be increased while keeping the aggregated bandwidth constant. This has similarities with oversampling and would result in a decrease in the condition number, thereby improving the SNR (and EVM), see Fig. 4.

Consequently, a conventional sampling system cannot re-

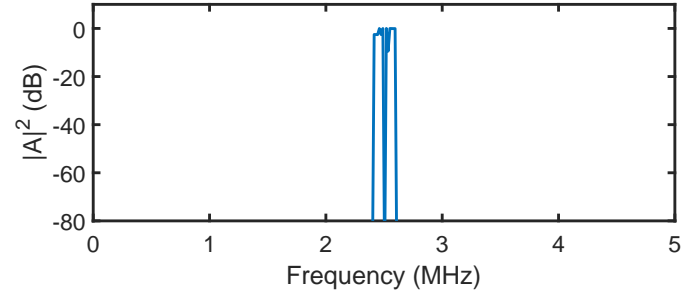

(a)

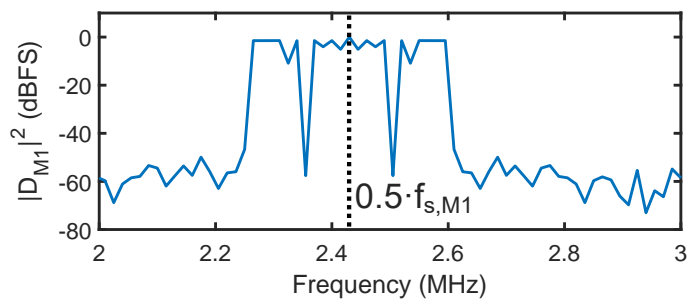

(b)

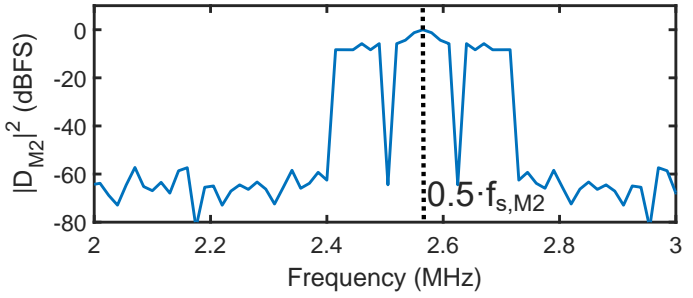

(c)

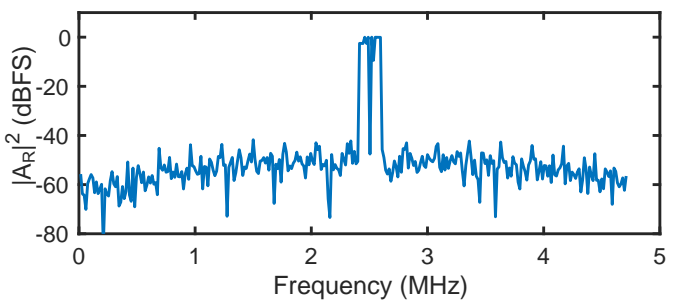

(d)

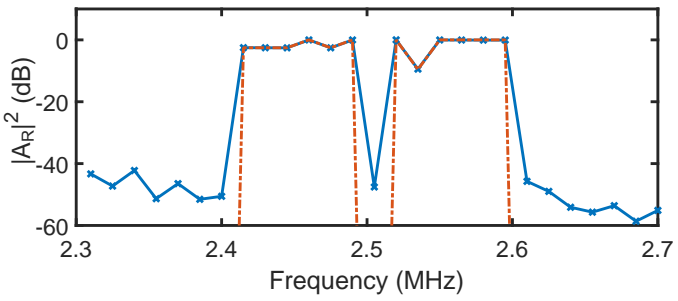

(e)

Fig. 12: Measured spectral snapshot of a single OFDM symbol out of a 200 symbol sequence with 12 sub-carriers centred at $2.505 \mathrm{MHz}$. (a) The spectrum of the $180 \mathrm{kHz}$ OFDM signal centred at $2.505 \mathrm{MHz}$. (b) Zoomed in DFT spectrum $\left|\mathbf{D}_{\mathbf{M}_{1}}\right|^{2}$ around the signal of interest when the input signal is sampled at $4.86 \mathrm{MS} / \mathrm{s}$. (c) Zoomed in DFT spectrum $\left|\mathbf{D}_{\mathbf{M}_{\mathbf{2}}}\right|^{2}$ around the signal of interest when the input signal is sampled at $5.13 \mathrm{MS} / \mathrm{s}$. (d) Reconstructed spectrum of the OFDM symbol by using the MRU algorithm. (e) Zoom-in of (d) showing both the measured (solid line) and ideal input (dash line) signals.

construct this (aliased) signal without changing the sample frequency. Even so, when the signal frequency changes or an additional bandpass signal is added the constraints on the sample rate to prevent any aliasing increases in conventional sampling. 


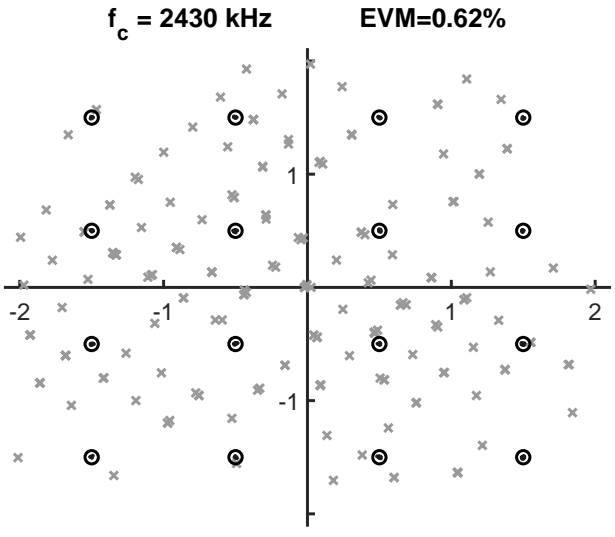

Fig. 13: Measured constellation diagram of the sub-carrier with the highest EVM for the OFDM signal in Fig.12. The circles are the ideal constellation points, the dots are the reconstructed results when using the MRU algorithm and the crosses are the reconstructed results of a single ADC.

Fig. 12(d) shows the output of the MRU algorithm and Fig. 12(e) shows a zoom in together with the ideal input signal. The reconstructed signal shows that the OFDM signal is indeed centred around $2.505 \mathrm{MHz}$ and the frequency ambiguity in the DFTs of $A D C_{1}$ and $A D C_{2}$ is resolved. The power level of the sub-carrier at $2.505 \mathrm{MHz}$ is $9.5 \mathrm{~dB}$ lower than the maximum measured sub-carrier power as it lies on the inner circle of the 16-QAM constellation in Fig.13. The effect of ADC noise and condition number is barely visible in Fig. 12(e): the measured and ideal input spectra lie on top of each other. The recovered spectra in Fig. 12(e) seems to approach the ideal input, however there are some errors which are hardly visible due to the logarithmic scale. The upper boundary for the errors is set by the accuracy of the ADC. The second most important factor of performance degradation is the propagation of errors due to the MRU algorithm, where the degradation is limited to the condition number $\kappa . \kappa$ is determined by the chosen sample frequencies and the selected sub-carriers.

To determine the effect of the MRU algorithm on the quality of the received OFDM signal, the EVM for every sub-carrier is determined. The RMS EVM over N-symbols for an M-ary signal constellation is calculated as:

$$
\mathrm{EVM}_{\mathrm{RMS}}=\frac{\sqrt{\frac{1}{N} \sum_{i=1}^{N}\left|S_{\text {ideal }, i}-S_{\text {meas }, i}\right|^{2}}}{\sqrt{\frac{1}{M} \sum_{i=1}^{M}\left|S_{\text {ideal }, i}\right|^{2}}}
$$

where $S_{\mathrm{ideal}, i}$ and $S_{\text {meas }, i}$ are respectively the ideal and measured vectors corresponding to the $i$ th symbol.

Fig. 13 shows the constellation before and after applying the MRU algorithm for the sub-carrier with the worst EVM due to quantization plus aliasing and Fig. 14 shows the EVM of all the sub-carriers in the OFDM signal measured over 200 symbols. The higher sub-carriers in Fig. 12 are corrupted by in-band interference due to aliasing, which prevents demodulation of the signal in a conventional way. It shows that the modulated data is recovered with an EVM lower than $0.62 \%$ ($44 \mathrm{~dB}$ ). To compare the effect of degradation due to the MRU algorithm alone, the EVM for the lower sub-carriers in Fig. 14

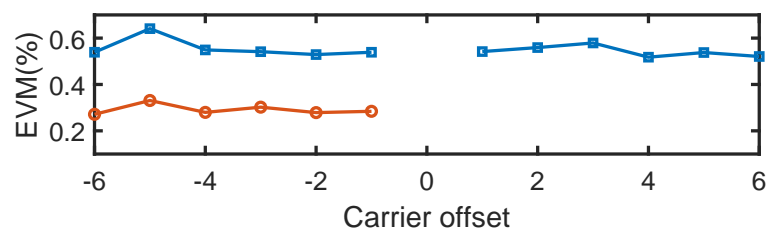

Fig. 14: Measured EVM over 200 symbols of each sub-carrier of the OFDM signal after demodulation by using the MRU algorithm (squares) and a single ADC (circles). For the single ADC only the EVM of the sub-carriers that can be successfully demodulated is shown.

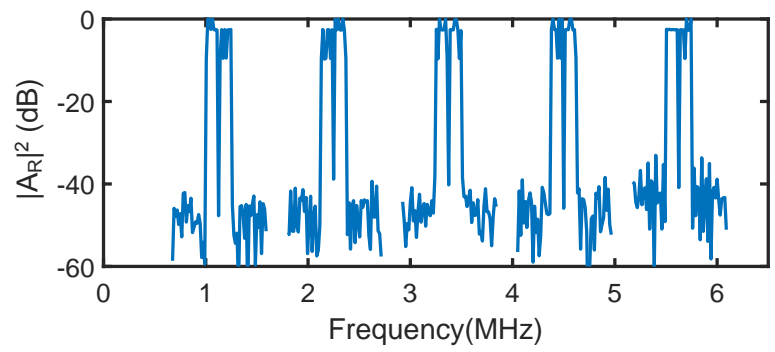

(a)

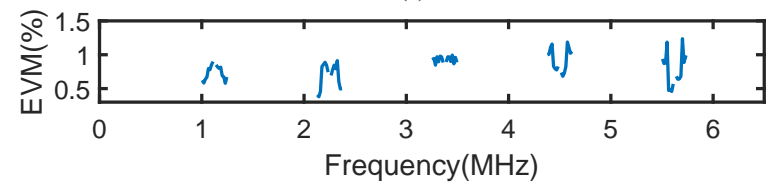

(b)

Fig. 15: (a) Sequentially measured spectra of a single OFDM symbol out of a 200 symbol sequence with the center frequency in different Nyquist zones. (b) EVM corresponding to the OFDM signal in different Nyquist zones.

is also determined. These lower sub-carriers in $\left|\mathbf{D}_{\mathbf{M}_{\mathbf{2}}}\right|^{2}$ are not corrupted by aliasing. The average EVM for these sub-carriers is $0.28 \%(-50 \mathrm{~dB})$. Using the MRU algorithm the signal can be demodulated at a cost of about $12 \mathrm{~dB}$ higher SNR compared to the case when there is no in-band interference. The $12 \mathrm{~dB}$ SNR degradation translates to $6 \mathrm{~dB}$ EVM degradation in case of no in-band interference, since $\mathrm{EVM}_{\mathrm{RMS}} \approx \mathrm{SNR}^{-\frac{1}{2}}[18]$. The measured $12 \mathrm{~dB}$ SNR degradation agrees with the simulated $12 \mathrm{~dB}$ SNR degradation as shown in Fig. 4.

\section{B. Reconstruction of aliased signal outside first Nyquist Zone}

The performance of the MRU algorithm when demodulating a single signal in higher Nyquist zone is demonstrated. The operating frequency of the ADCs is lowered compared to the previous measurement to allow sampling in multiple Nyquist zones, since the input bandwidth of the used low power ADCs is limited to $5 \mathrm{MHz}$. The sample rates are set to $1.17 \mathrm{MS} / \mathrm{s}\left(M_{1}=78\right)$ and $0.93 \mathrm{MS} / \mathrm{s}\left(M_{2}=62\right)$ for $A D C_{1}$ and $A D C_{2}$ respectively. The OFDM signal from the previous measurements is used and is centred around $N \cdot 1.125 \mathrm{MHz}$ in each sequential measurement where $1 \leq N \leq 5$.

Fig. 15(a) shows the sequentially reconstructed spectra up to $5.625 \mathrm{MHz}$ using the MRU algorithm; Fig. 15(b) shows the EVM of the OFDM sub-carriers over all center frequencies in the different Nyquist zones. These figures show that the input signal frequencies in multiple Nyquist zones were recovered. 
C. Reconstruction of two aliased signals from distinct Nyquist zones

To demonstrate the ability of the MRU algorithm to concurrently solve frequency ambiguity and determine the subcarrier frequency before sampling, a dual-band OFDM signal that is spread over different Nyquist-zones is used as an input signal. After sampling the two OFDM bands become indistinguishable from each other, however MRU can resolve this allowing individual reconstruction of the two bands and determining the EVM.

The center frequency of the first OFDM band is $1.8 \mathrm{MHz}$ $(\mathrm{BW}=180 \mathrm{kHz})$ and that of the second OFDM band is 7.05 $\mathrm{MHz}(\mathrm{BW}=750 \mathrm{kHz})$, resulting in aliasing in both DFT spectra. The sample rates for $A D C_{1}$ and $A D C_{2}$ are set to 2.43 $\mathrm{MS} / \mathrm{s}\left(M_{1}=162\right)$ and $2.565 \mathrm{MS} / \mathrm{s}\left(M_{2}=171\right)$. The $B W_{\max }$ of this system is $2.43 \mathrm{MHz}$ (see (17)). Since the aggregated BW of the input signal $(0.93 \mathrm{MHz})$ is smaller than $2.43 \mathrm{MHz}$, the MRU algorithm can solve the frequency ambiguity caused by sampling allowing reconstruction of both OFDM bands. The aliasing matrix $\mathbf{P}$ is chosen such that it covers all the sub-carriers in the dual-band OFDM signal.

The measured frequency spectra of a single OFDM symbol by both ADCs is shown in Fig. 16(a) and 16(b) in blue. The original input spectra are added in solid grey and dashed black lines, and any overlap between the two input spectra would impact the demodulation performance significantly. In Fig. 16 the ratio $P_{R}$ between the average power of the first and second band-pass signal is set to $-20 \mathrm{~dB}$ for illustration purposes. Fig. 16(c) shows the recovered frequency spectrum after applying the MRU algorithm, clearly showing that the frequency ambiguity is removed. Since the MRU algorithm does not solve the frequency bands between the two OFDM bands there is no data corresponding to those frequencies. Fig. 16(d) shows the zoom-in around the first band for a single OFDM symbol and Fig. 16(e) shows the zoom-in of the second band for a single OFDM symbol. Compared to a conventional sample system that would sample at $2 f_{\text {span }} \approx 15$ $\mathrm{MHz}, \mathrm{MRU}$ reduces the individual sample rates $(2.43 \mathrm{MS} / \mathrm{s}$ and $2.565 \mathrm{MS} / \mathrm{s}$ ) and output data rates by a factor 6 .

Fig. 17 shows the EVM of the demodulated dual-band OFDM signal with and without the MRU algorithm. The power ratio $P_{R}$ between the two band-pass signals is swept from $-18 \mathrm{~dB}$ to $18 \mathrm{~dB}$ in steps of $6 \mathrm{~dB}$ and the signal is scaled in such a way that the total dual-band signal range exactly fits the full-scale range of the ADC to minimize quantization errors and to also avoid distortion due to clipping. For low power ratios the increase in EVM by using the MRU algorithm is due to the fact that the (desired) signal amplitude is also decreased to prevent clipping in the $\mathrm{ADC}$, thereby reducing the SNR of the input.

From Fig. 17 it can be concluded that by using the MRU algorithm, EVM can be improved by a maximum of $42 \mathrm{~dB}$ for this setup, allowing successful demodulation and characterization of the wanted signal in the presence of strong signals which after sampling can become in-band, e.g. intermodulation products. For a power ratio of $-18 \mathrm{~dB}$ the desired signal can still be demodulated with an EVM of $-27 \mathrm{~dB}$.

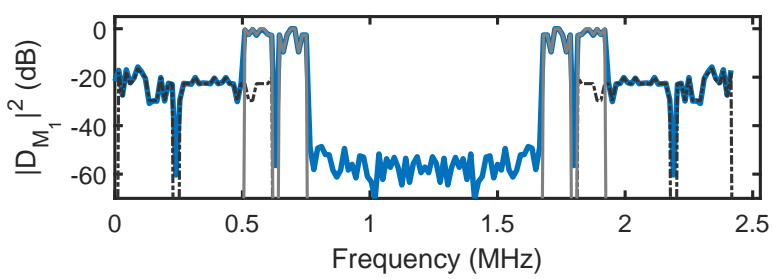

(a)

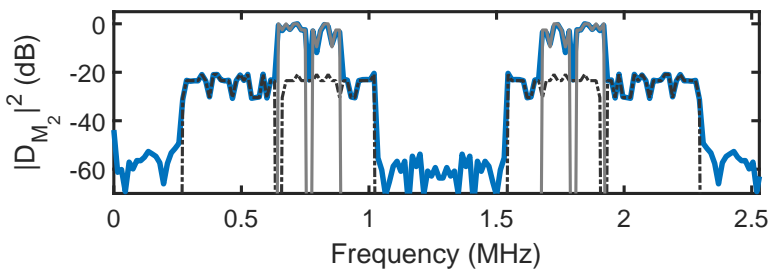

(b)

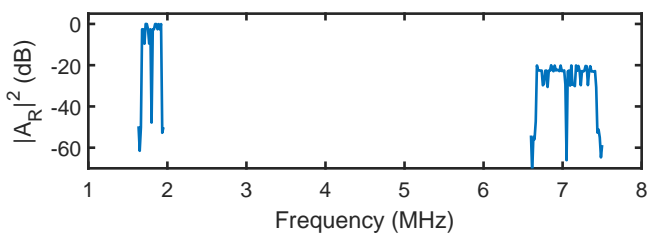

(c)

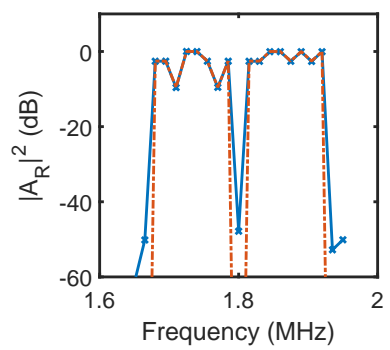

(d)

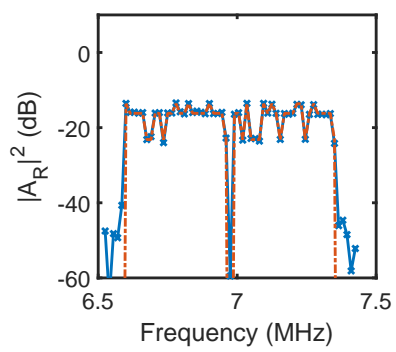

(e)
Fig. 16: Measured spectra of the dual-band OFDM signal for a single OFDM symbol out of a 200 symbol sequence. (a) $2.43 \mathrm{MS} / \mathrm{s}$ DFT spectrum $\left|\mathbf{D}_{\mathbf{M}_{1}}\right|^{2}$ (solid blue) with the ideal input signals (dash black and solid grey line). (b) 2.565 MS/s DFT spectrum $\left|\mathbf{D}_{\mathbf{M}_{2}}\right|^{2}$ (solid blue) with the ideal input signals (dash black and solid grey line). (c) Recovered split-bandwidth spectrum. The dual-band OFDM signal is separated allowing independent reconstruction. (d) and (e) shows the zoom-in of (c) with both the measured (solid line) and the ideal input (dash line).

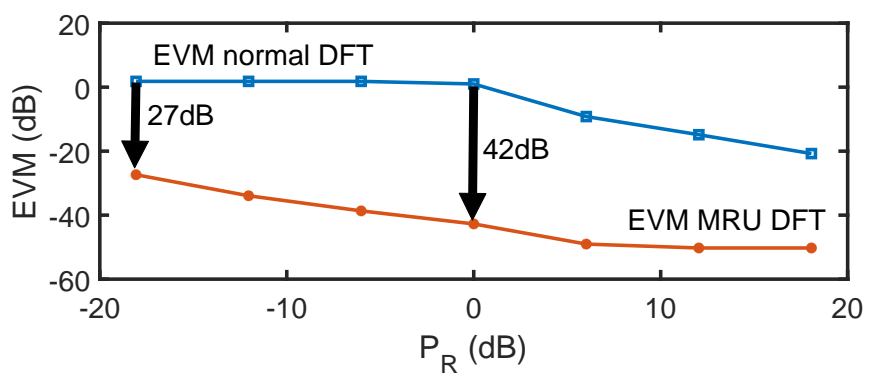

Fig. 17: EVM of a demodulated OFDM signal in the presence of another OFDM signal with and without the MRU algorithm. The maximum improvement in EVM by using our MRU algorithm is $42 \mathrm{~dB}$ and is limited by the setup. The lower limit in EVM of the MRU algorithm is due to the SNR of the ADC and the SNR degradation due to the condition number. The total input signal is scaled to fit within the ADCs dynamic range to avoid clipping. 


\section{CONCLUSION}

A novel multi-rate unaliasing algorithm (MRU) that solves frequency ambiguity due to aliasing of (sub-)sampled multiband signals with an orthogonal frequency basis is presented. Sampling the input signal at two different sample frequencies that are non-integer multiples of one another results in two distinct systems of linear equations. Combining them increases the overall rank of the system of linear equations allowing the MRU algorithm to solve frequency ambiguity due to sampling. Additionally the MRU algorithm allows reconstruction of the input signal spectrum, which can be (sparsely) spread over a wide bandwidth equal to the least common multiple of the two sample frequencies. Furthermore MRU allows reconstruction of an aliased signal with an aggregated bandwidth close to the Nyquist-Shannon limit. The MRU algorithm can solve for frequency ambiguity in multiple Nyquist zones by using two different clock rates and some additional digital signal processing. A demonstrator setup comprising of two 10 bit ADCs clocked at two different sample rates is used to demonstrate the effectiveness of the MRU algorithm in the reconstruction of aliased signals.

\section{APPENDIX A}

\section{NUMERICAL EXAMPLE OF THE MRU ALGORITHM}

This appendix gives a numerical example of the algorithm described in this paper. In this example the set of modulated sub-carriers $S$ in the input signal $x(t)$ is chosen as:

$$
S=\{0,1,3,5\}
$$

and the set $A$ containing the complex value coefficients corresponding to the sub-carriers in $S$ are chosen to be:

$$
A=\{0, j 1, j 2, j 3\}
$$

where $j^{2}=-1$. The sets $S$ and $A$ respectively describe the spectral and complex value (magnitude and phase) content of the input signal $x(t)$ in equation (1). The input signal is then sampled at two different (non-integer multiples) frequencies $M_{1} f_{o}$ and $M_{2} f_{o}$, where in this example $M_{1}=4$ and $M_{2}=5$. Sampling for a duration of $\frac{1}{f_{o}}$ and performing a DFT on the sampled data results in the DFT vectors $\mathbf{D}_{\mathbf{M} 1}$ and $\mathbf{D}_{\mathbf{M} 2}$ :

$$
\begin{gathered}
D_{M 1}=\left[\begin{array}{llll}
0 & j 2 & 0 & -j 2
\end{array}\right]^{\top} \\
D_{M 2}=\left[\begin{array}{lllll}
0 & j 1 & -j 2 & j 2 & -j 1
\end{array}\right]^{\top} .
\end{gathered}
$$

Combining the results of $D_{M 1}$ and $D_{M 2}$ into the vector $D_{c}$ results in:

$$
D_{c}=\left[\begin{array}{lllllllll}
0 & j 2 & 0 & -j 2 & 0 & j 1 & -j 2 & j 2 & -j 1
\end{array}\right]^{\top} .
$$

In order to reconstruct the complex values of the sub-carriers in the input signal, the MRU algorithm requires both the combined DFT vector $\mathbf{D}_{\mathbf{c}}$ and the aliasing pattern, $\mathbf{P}_{\mathbf{c}}$ (16).

The matrix $\mathbf{P}_{\mathbf{c}}$ is pre-calculated based on the sub-carriers in $S . \mathbf{P}_{\mathbf{c}}$ describes the contribution of the complex values in $A$ belonging to the sub-carriers in $S$ to the DFT bins in $\mathbf{D}$. Using (11) and (12) the values of each of the elements in the matrix $\mathbf{P}$ are calculated. The elements in $\mathbf{P}$ are equal to 1 if
$S_{l}=k+r M$ in case of the even columns and $S_{l}=-k+r M$ in case of the odd columns where $k$ is the row number and $0 \leq l<|S|$. The aliasing pattern $\mathbf{P}_{\mathbf{M} 1}$ corresponding to a sample rate of $M_{1} f_{o}=4 f_{o}$ is:

$$
P_{M 1}=\left(\begin{array}{cccccccc}
1 & 1 & 0 & 0 & 0 & 0 & 0 & 0 \\
0 & 0 & 1 & 0 & 0 & 1 & 1 & 0 \\
0 & 0 & 0 & 0 & 0 & 0 & 0 & 0 \\
0 & 0 & 0 & 1 & 1 & 0 & 0 & 1
\end{array}\right)
$$

As described in Section III, the even columns in (33) contain information about the contribution of $A$ to $\mathbf{D}$ and the odd columns describe the contribution of $A^{*}$ to $\mathbf{D}$. To illustrate how the matrix $\mathbf{P}_{\mathbf{c}}$ links $A$ to $\mathbf{D}$ we use the second row of (33) in (10) resulting in :

$$
\begin{aligned}
D_{M 1}[1]= & P_{M 1}[0] A_{0}+P_{M 1}[1] A_{0}^{*}+P_{M 1}[2] A_{1}+ \\
& P_{M 1}[3] A_{1}^{*}+P_{M 1}[4] A_{2}+P_{M 1}[5] A_{2}^{*}+ \\
& P_{M 1}[6] A_{3}+P_{M 1}[7] A_{3}^{*} \\
= & j 1-j 2+j 3=j 2
\end{aligned}
$$

which matches $D_{M 1}[1]$ in (30).

The aliasing pattern $P_{M 2}$ corresponding to a sample rate of $M_{2} f_{o}=5 f_{o}$ is:

$$
P_{M 2}=\left(\begin{array}{cccccccc}
1 & 1 & 0 & 0 & 0 & 0 & 0 & 0 \\
0 & 0 & 1 & 0 & 0 & 0 & 0 & 0 \\
0 & 0 & 0 & 0 & 1 & 0 & 0 & 1 \\
0 & 0 & 0 & 0 & 0 & 1 & 1 & 0 \\
0 & 0 & 0 & 1 & 0 & 0 & 0 & 0
\end{array}\right)
$$

The combined pattern matrix $P_{c}$ is calculated by using (33) and (35) in (15), resulting in:

$$
P_{c}=\left(\begin{array}{llllllll}
1 & 1 & 0 & 0 & 0 & 0 & 0 & 0 \\
0 & 0 & 1 & 0 & 0 & 1 & 1 & 0 \\
0 & 0 & 0 & 0 & 0 & 0 & 0 & 0 \\
0 & 0 & 0 & 1 & 1 & 0 & 0 & 1 \\
1 & 1 & 0 & 0 & 0 & 0 & 1 & 1 \\
0 & 0 & 1 & 0 & 0 & 0 & 0 & 0 \\
0 & 0 & 0 & 0 & 0 & 1 & 0 & 0 \\
0 & 0 & 0 & 0 & 1 & 0 & 0 & 0 \\
0 & 0 & 0 & 1 & 0 & 0 & 0 & 0
\end{array}\right)
$$

Calculating the left-inverse of $\mathbf{P}_{\mathbf{c}}$ results in $\mathbf{P}_{\mathbf{c}}^{-\mathbf{1}}$ (rounded to second decimal in this example), which together with $\mathbf{D}_{\mathbf{c}}$ enables the reconstruction of the complex values $A$ (magnitude and phase) corresponding to the sub-carriers described by $S$. The reconstructed complex value vector $\mathbf{A}_{\mathbf{R}}$ using $\mathbf{P}_{\mathbf{c}}^{-\mathbf{1}}$ and $\mathbf{D}_{\mathbf{c}}$ can be calculated from (37).

\section{APPENDIX B \\ IQ EXTENSION OF THE MRU ALGORITHM}

Conventional IQ demodulation systems already use two ADCs, one in each of the I and Q paths. This section will derive the algorithm such that MRU can be used in an IQ system. The system overview is given in Fig. 18. 


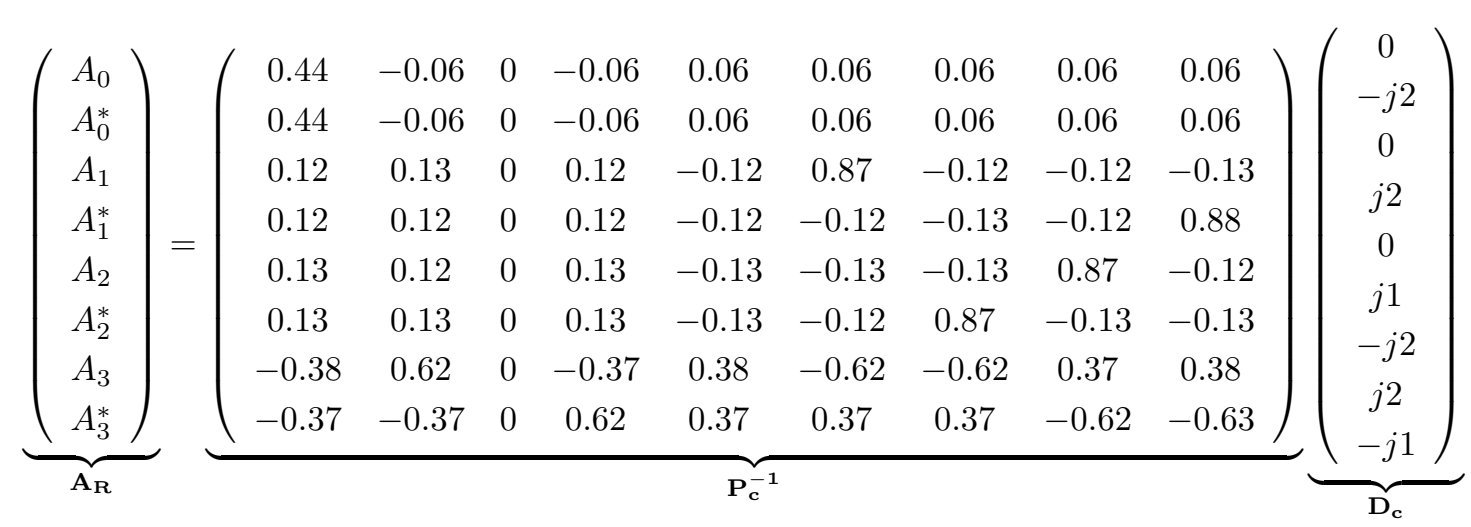

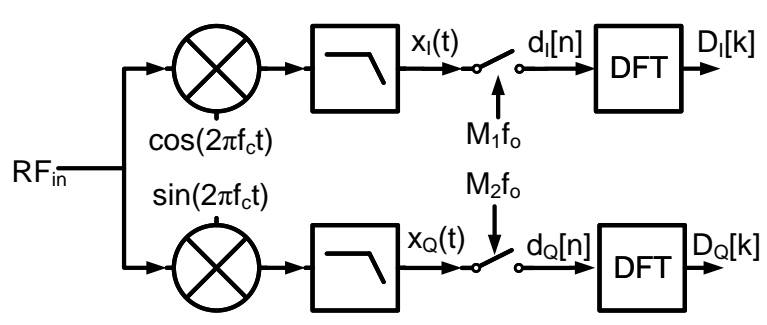

Fig. 18: MRU IQ system overview. The lowpass filters are only used to filter out the high frequency content and are not used as anti-aliasing filters.

The RF-input signal can be described by

$$
x_{R F}(t)=\sum_{l \in L}\left(A_{l} e^{j 2 \pi\left(f_{c}+S_{l} f_{o}\right) t}+A_{l}^{*} e^{-j 2 \pi\left(f_{c}+S_{l} f_{o}\right) t}\right),
$$

where $f_{c}$ is the bandpass center frequency and $S_{l} \in \mathbb{Z}$. Down conversion by respectively $\cos \left(2 \pi f_{c} t\right)$ and $\sin \left(2 \pi f_{c} t\right)$ and filtering the up-conversion products results in the baseband I and Q signals give by

$$
x_{I}(t)=\sum_{l \in L}\left(A_{l} e^{j 2 \pi S_{l} f_{o} t}+A_{l}^{*} e^{-j 2 \pi S_{l} f_{o} t}\right)
$$

and $x_{Q}(t)=-j x_{I}(t)$.

Sampling the analog signals $x_{I}(t)$ and $x_{Q}(t)$ results in the two data-streams $d_{I}[n]$ and $d_{Q}[n]$. Performing a DFT on both $d_{I}[n]$ and $d_{Q}[n]$ results in the two dataset

$$
\begin{array}{r}
D_{I}[k]=\sum_{l \in L}\left(\sum _ { r = - \infty } ^ { \infty } \left(A_{l} \delta\left(S_{l}-k-r M\right)+\right.\right. \\
\left.\left.A_{l}^{*} \delta\left(S_{l}+k-r M\right)\right)\right),
\end{array}
$$

which describe the elements of the complex DFT vectors $\mathbf{D}_{\mathbf{I}}$ and $\mathbf{D}_{\mathbf{Q}}$ and $D_{Q}[k]=-j D_{I}[k]$.

The matrix $\mathbf{P}_{\mathbf{I}}$ describing the aliasing pattern of the input signal to $\mathbf{D}_{\mathbf{I}}$ is equal to (11) and (12), since they both describe the in-phase aliasing pattern. The matrix $\mathbf{P}_{\mathbf{Q}}$ describing the quadrature aliasing pattern is derived from $D_{Q}[k]$ in a similar way as (11) and (12) are derived and is given by

$$
P_{Q}[k, 2 l]=\sum_{r=-\infty}^{\infty} \delta\left(S_{l}-k-r M\right)
$$

$$
P_{Q}[k, 2 l+1]=-\sum_{r=-\infty}^{\infty} \delta\left(S_{l}+k-r M\right)
$$

The matrices $\mathbf{P}_{\mathbf{c}}$ and $\mathbf{D}_{\mathbf{c}}$ are formed similar to (15) and (14) by combining $\mathbf{P}_{\mathbf{I}}$ with $\mathbf{P}_{\mathbf{Q}}$ and $\mathbf{P}_{\mathbf{I}}$ with $\mathbf{P}_{\mathbf{Q}}$ resulting in

$$
\mathbf{P}_{\mathbf{c}}=\left[\begin{array}{c}
\mathbf{P}_{\mathbf{I}} \\
\mathbf{P}_{\mathbf{Q}}
\end{array}\right], \mathbf{D}_{\mathbf{c}}=\left[\begin{array}{c}
\mathbf{D}_{\mathbf{I}} \\
\pm j \mathbf{D}_{\mathbf{Q}}
\end{array}\right] \text {. }
$$

where the sign of $\mathbf{D}_{\mathbf{Q}}$ determines if the MRU algorithm should solve for the upper (+) or lower sideband (-). The final reconstruction is analog to the conventional case by using (16).

A simulation is performed to demonstrate MRU in an IQ system. Fig. 19(a) shows the RF-input signal. It consists of two $1.5 \mathrm{MHz}$ OFDM signals centred at $2.4 \mathrm{GHz}$ and $2.4036 \mathrm{GHz}$. The RF signal is down-converted, filtered and sampled. Fig. 19 (b) and (c) shows the resulting DFTs $\mathbf{D}_{\mathbf{I}}$ and $\mathbf{D}_{\mathbf{Q}}$ of the sampled datastreams $d_{I}[n]$ and $d_{Q}[n]$. Finally Fig. 19(d) and (e) show a zoom-in of the separately reconstructed bands with an overlay of the original input spectrum, demonstrating that the input and output spectra are equal.

Any mismatch between the I and Q paths degrades the accuracy of the signal demodulation, where commonly occurring mismatch in IQ systems are gain and phase mismatch. Fig. 20 shows the simulated results for the gain mismatch $A_{\epsilon}$ normalized to the ideal gain (e.g. $10^{-2}=1 \%$ ) and phase mismatch $\phi_{\epsilon}$ normalized to $2 \pi$ for both a conventional oversampled IQ system and the MRU algorithm. Compared to the conventional case the MRU algorithm degrades the EVM by $1 \mathrm{~dB}$ and $1.5 \mathrm{~dB}$ for phase and gain mismatch respectively. The degradation is independent on the amount of mismatch, where the degradation only depends on the condition number of the matrix $\mathbf{D}_{\mathbf{c}}$ (see section V-A).

\section{APPENDIX C}

\section{CHARACTERIZATION OF HARMONICS IN AN RF-PA}

Another use case for the MRU algorithm is the characterization of the harmonics of an RF-waveform to improve the reliability and pre-distortion in an RF-PA as mentioned in Section I. The work in [5] uses 8 equally spaced samples over a single period of the RF-waveform, allowing characterization of the DC component and the first 3 harmonics. However, when the RF-waveform contains more harmonics aliasing will occur. Down-mixing with a square wave at $f_{o}$ results in the 


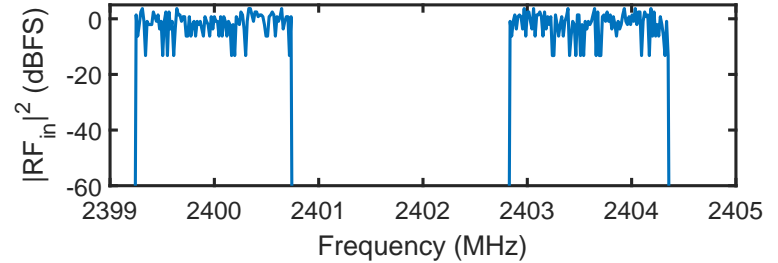

(a)

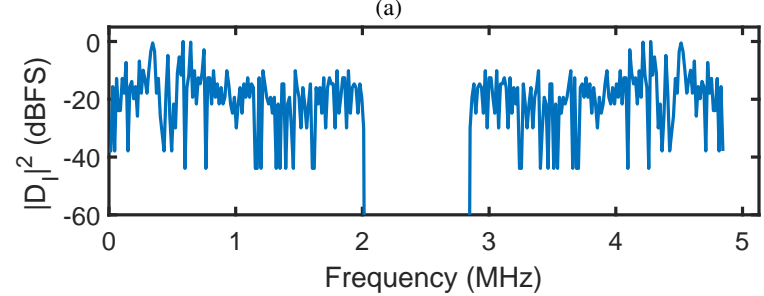

(b)

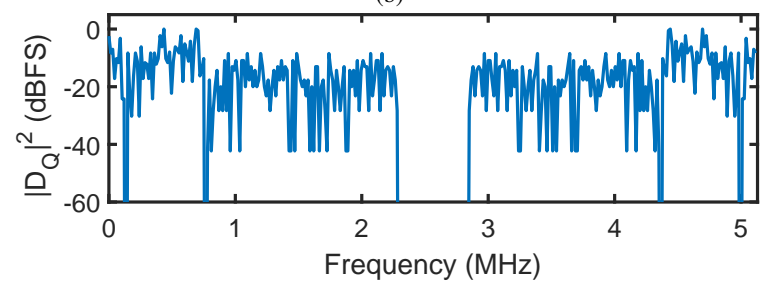

(c)

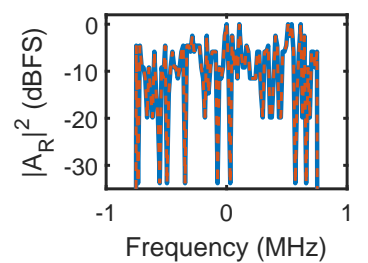

(d)

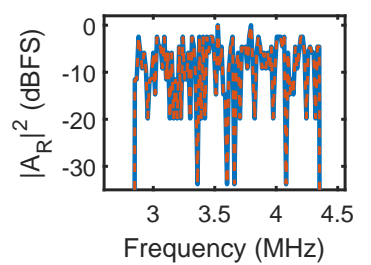

(e)
Fig. 19: (a) RF-input spectrum. Measured spectra of the dual-band OFDM signal after down-conversion for a single symbol. (b) $4.86 \mathrm{MS} / \mathrm{s}$ DFT spectrum $\left(\left|D_{I}\right|^{2}\right)$ of the I-path. (c) $5.13 \mathrm{MS} / \mathrm{s}$ DFT spectrum $\left(\left|D_{Q}\right|^{2}\right)$ of the Q-path. (d) and (e) shows the zoom-in of the recovered signal with both the measured (solid line) and the ideal input (dash line).

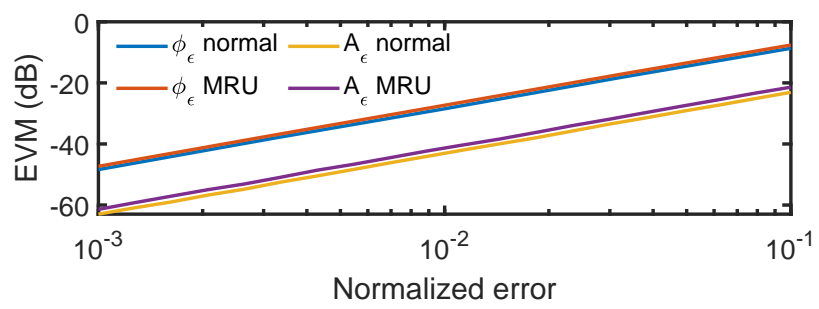

Fig. 20: Simulated EVM due to gain and phase mismatch in an IQ system. The results are for both a conventional oversampled system and the proposed MRU algorithm. $A_{\epsilon}$ is the normalized gain mismatch (e.g. $10^{-2}=1 \%$ ) and the phase mismatch $\phi_{\epsilon}$ is normalized to $2 \pi$.

harmonics becoming indistinguishable, where mixing with a frequency offset can result in frequency pulling. To prevent aliasing the sample rate can be increased, but this would increase the system clock rate which might not be feasible at all times. MRU can increase the number of distinguishable harmonics by adding another sampler at a lower sample rate, such as 7 samples per period.

Fig. 21 (a) shows an RF-waveform with $f_{o}=2.5 \mathrm{GHz}$ and the resulting samples when sampling at rates of $20 \mathrm{GS} / \mathrm{s}$
$\left(M_{1}=8,{ }^{\prime} \square\right.$ ') and $17.5 \mathrm{GS} / \mathrm{s}\left(M_{2}=7\right.$, , $^{\prime}$ ') $)$. Fig. 21 (b) and (c) show the aliased DFT spectra of the RF-input signal when sampling at $20 \mathrm{GS} / \mathrm{s}$ and $17.5 \mathrm{GS} / \mathrm{s}$ respectively. Fig. 21 (d) shows the RF-input spectrum (line) and the reconstructed RF-spectrum (o) using MRU. By using MRU the number of harmonics that can be characterized is increased from 3 to 6 . In contrast, a conventional system to characterize these harmonics would require a sample rate higher than $30 \mathrm{GS} / \mathrm{s}$.

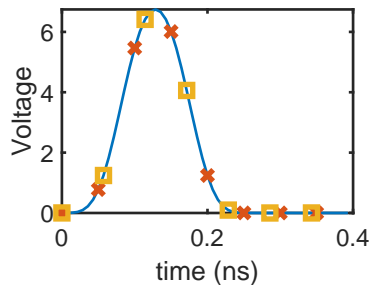

(a)

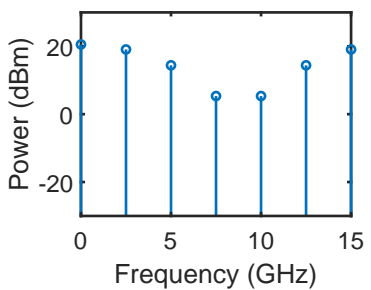

(c)

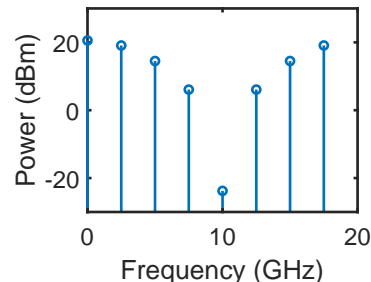

(b)

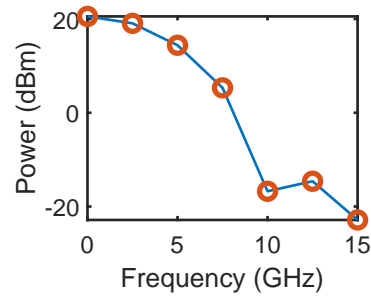

(d)
Fig. 21: (a) Characterization of a $f_{o}=2.5 \mathrm{GHz}$ RF-waveform in an RF-PA. The ' $\square$ ' and ' $\times$ ' indicate the sampled signal for $M_{1}=8$ and $M_{2}=7$ respectively. (b) and (c) are the DFT spectra $\left|\mathbf{D}_{1}\right|^{2}$ and $\left|\mathbf{D}_{2}\right|^{2}$ for $M_{1}=8$ and $M_{2}=7$ samples per period respectively. (d) Input RFwaveform spectrum (line) and the reconstructed spectrum $\left|A_{R}\right|^{2}$ (o).

\section{REFERENCES}

[1] R. B. Sanderson, J. B. Y. Tsui, and N. A. Freese, "Reduction of aliasing ambiguities through phase relations," IEEE Transactions on Aerospace and Electronic Systems, vol. 28, no. 4, pp. 950-956, Oct 1992.

[2] D. W. Tufts and H. Ge, "Digital estimation of frequencies of sinusoids from wide-band under-sampled data," in 1995 International Conference on Acoustics, Speech, and Signal Processing, vol. 5, May 1995, pp. $3155-3158$ vol.5.

[3] X. . Xia, "An Efficient Frequency-Determination Algorithm from Multiple Undersampled Waveforms," IEEE Signal Processing Letters, vol. 7, no. 2, pp. 34-37, Feb 2000.

[4] P. P. Vaidyanathan and P. Pal, "Sparse Sensing With Co-Prime Samplers and Arrays," IEEE Transactions on Signal Processing, vol. 59, no. 2, pp. 573-586, Feb 2011.

[5] M. Huiskamp, A. J. Annema, and B. Nauta, "A Delay Spread Cancelling Waveform Characterizer for RF Power Amplifiers," IEEE Transactions on Circuits and Systems II: Express Briefs, vol. 65, no. 12, pp. 1834$1838,2018$.

[6] S. Weinstein and P. Ebert, "Data Transmission by Frequency-Division Multiplexing Using the Discrete Fourier Transform," IEEE Transactions on Communication Technology, vol. 19, no. 5, pp. 628-634, October 1971.

[7] C. E. Shannon, "Communication in the Presence of Noise," Proceedings of the IRE, vol. 37, no. 1, pp. 10-21, Jan 1949.

[8] R. G. Vaughan, N. L. Scott, and D. R. White, "The Theory of Bandpass Sampling," IEEE Transactions on Signal Processing, vol. 39, no. 9, pp. 1973-1984, Sep. 1991.

[9] D. M. Akos, M. Stockmaster, J. B. Y. Tsui, and J. Caschera, "Direct Bandpass Sampling of Multiple Distinct RF Signals," IEEE Transactions on Communications, vol. 47, no. 7, pp. 983-988, July 1999.

[10] J. Gaskell, Linear Systems, Fourier Transforms, and Optics. New York: Wiley, 1978.

[11] M. Pelgrom, Analog-to-Digital Conversion. Springer, 2010. 
[12] B. Razavi, "Problem of timing mismatch in interleaved ADCs," in Proceedings of the IEEE 2012 Custom Integrated Circuits Conference, Sep. 2012, pp. 1-8.

[13] M. El-Chammas and B. Murmann, "General Analysis on the Impact of Phase-Skew in Time-Interleaved ADCs," IEEE Transactions on Circuits and Systems I: Regular Papers, vol. 56, no. 5, pp. 902-910, May 2009.

[14] N. Sinha, M. Rachid, and S. Pamarti, "A sharp programmable passive filter based on filtering by Aliasing," in 2015 Symposium on VLSI Circuits (VLSI Circuits), June 2015, pp. C58-C59.

[15] B. J. Thijssen, E. A. M. Klumperink, P. Quinlan, and B. Nauta, "A 0.06-3.4-MHz 92- $\mu$ W Analog FIR Channel Selection Filter With Very Sharp Transition Band for IoT Receivers," IEEE Solid-State Circuits Letters, vol. 2, no. 9, pp. 171-174, 2019

[16] H. S. Bindra, A. J. Annema, G. Wienk, B. Nauta, and S. M. Louwsma, "A 4MS/s 10b SAR ADC with integrated Class-A buffers in $65 \mathrm{~nm}$ CMOS with near rail-to-rail input using a single $1.2 \mathrm{~V}$ supply," in 2019 IEEE Custom Integrated Circuits Conference (CICC), 2019, pp. 1-4.

[17] H. S. Bindra, A. J. Annema, S. M. Louwsma, and B. Nauta, "A 0.2 $8 \mathrm{MS} / \mathrm{s} 10 \mathrm{~b}$ flexible SAR ADC achieving $0.35-2.5 \mathrm{fJ} / \mathrm{conv}$-step and using self-quenched dynamic bias comparator," in 2019 Symposium on VLSI Circuits, June 2019, pp. C74-C75.

[18] A. Georgiadis, "Gain, phase imbalance, and phase noise effects on error vector magnitude," IEEE Transactions on Vehicular Technology, vol. 53 , no. 2, pp. 443-449, March 2004.

[19] "Evolved Universal Terrestrial Radio Access (E-UTRA); User Equipment (UE) radio transmission and reception," vol. 3GPP TS 36.101 version 15.8.0 Release 15, October 2018. [Online]. Available: https://www.etsi.org/deliver/etsi_ts/136100_136199/136101/15.08. 00_60/ts_136101v150800p.pdf.

[20] B. Jann, G. Chance, A. G. Roy, A. Balakrishnan, N. Karandikar, T. Brown, X. Li, B. Davis, J. L. Ceballos, N. Tanzi, K. Hausmann, H. Yoon, Y. Huang, A. Freiman, B. Geren, P. Pawliuk, and W. Ballantyne, "21.5 A 5G Sub-6GHz Zero-IF and mm-Wave IF Transceiver with MIMO and Carrier Aggregation," in 2019 IEEE International SolidState Circuits Conference - (ISSCC), Feb 2019, pp. 352-354

[21] J. Lee, S. Han, J. Lee, B. Kang, J. Bae, J. Jang, S. Oh, J. Chang, S. Kang, K. Y. Son, H. Lim, D. Jeong, I. Jong, S. Baek, J. H. Lee, R. Ni, Y. Zuo, C. Yao, S. Heo, T. B. Cho, and I. Kang, "A Sub-6-GHz 5G New Radio RF Transceiver Supporting EN-DC With 3.15-Gb/s DL and 1.27-Gb/s UL in 14-nm FinFET CMOS," IEEE Journal of Solid-State Circuits, vol. 54, no. 12, pp. 3541-3552, Dec 2019.

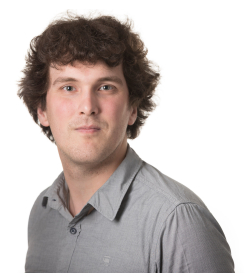

Maikel Huiskamp received the B.Sc and M.Sc degree (cum laude) in electrical engineering from the University of Twente, Enschede, The Netherlands, in 2012 and 2015, respectively. He is currently pursuing the $\mathrm{Ph} . \mathrm{D}$. degree with the Integrated Circuit Design Group, University of Twente, The Netherlands. His current research interests include sampling systems and efficient power amplifiers for RF applications.

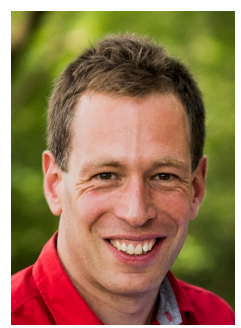

Mark S. Oude Alink (S'09-M'14-SM'19) was born in Hengelo, the Netherlands, in 1984. He received M.Sc. degrees in electrical engineering and computer science in 2008 (both cum laude) and a Ph.D. degree in 2013 (cum laude), from the University of Twente, Enschede, the Netherlands. He is a recipient of the prestigious Else Kooi award. After several years in industry as system and RFIC design engineer, he returned to his alma mater as assistant professor within the IC-Design group. His research focuses on low-power and digitally-assisted analog and RF circuits and systems. He has been serving on the Technical Program committee of the Custom Integrated Circuits Conference (CICC) since 2018, and is Guest Editor for the CICC Special Issue in the Journal of Solid-State Circuits.

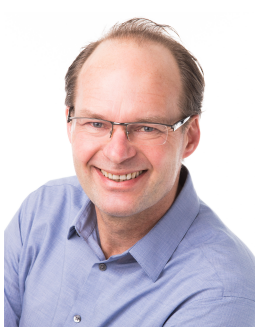

Bram Nauta (M'91-SM'03-F'08) was born in Hengelo, The Netherlands, in 1964. He received the M.Sc. degree (cum laude) in electrical engineering and the Ph.D. degree in analog CMOS filters for very high frequencies from the University of Twente, Enschede, The Netherlands, in 1987 and 1991, respectively.

In 1991, he joined the Mixed-Signal Circuits and Systems Department of Philips Research, Eindhoven The Netherlands. In 1998, he joined the University of Twente, where he has been a Chair of the EE Department since 2016, and currently a Distinguished Professor and heading the IC Design Group. His current research interests include high-speed analog CMOS circuits, software defined radio, cognitive radio, and beamforming.

Dr. Nauta is a member of the Royal Netherlands Academy of Arts and Sciences. He was a co-recipient of the ISSCC 2002 and 2009 "Van Vessem Outstanding Paper Award" and the "Simon Stevin Meester" Award (500.000), in 2014, the largest Dutch National Prize for achievements in technical sciences. He is currently the President of the IEEE Solid-State Circuits Society for 2018 to 2019 term. He served as the Editor-in-Chief of the IEEE Journal of Solid-State Circuits (JSSC) from 2007 to 2010 and the 2013 Program Chair of the International Solid State Circuits Conference.

Also, he served as Associate Editor of the IEEE TRANSACTIONS ON CIRCUITS AND SYSTEMS II from 1997 to 1999, and the IEEE JSSC from 2001 to 2006. He was in the Technical Program Committee of the Symposium on VLSI Circuits from 2009 to 2013 and is in the Steering Committee and Programme Committee of the European Solid State Circuit Conference. He served as a Distinguished Lecturer of the IEEE.

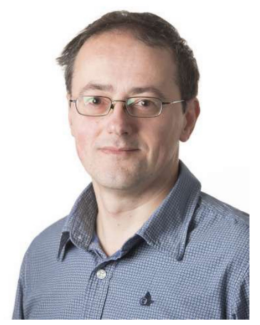

Anne-Johan Annema (M'00) received the M.Sc degree in electrical engineering and Ph.D. degree from the University of Twente, Enschede, The Netherlands, in 1990 and 1994, respectively.

In 1995, he joined the Semiconductor Device Architecture Department, Philips Research, Eindhoven, The Netherlands, where he was involved in a number of physics-electronics-related ranging from low-power low-voltage circuits, fundamental limits on analog circuits related to process technologies, and high voltage in baseline CMOS to feasibility research of future CMOS processes for analog circuits. Since 2000, he has been with the Integrated Circuit Design Group, Department of Electrical Engineering, University of Twente where he is associate professor and program director. He is also a part-time Consultant in industry and a co-founder of ChipDesignWorks, Twente. His current research interests include physics, analog, and mixed-signal electronics, RF power and deep submicrometer technologies and their joint feasibility aspects.

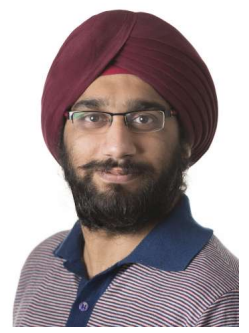

Harijot Singh Bindra (S'16) received the B.Tech. degree in electronics and communication engineering from Punjabi University, Patiala, India, in 2008, and the M.Tech. degree in VLSI design from the IIT, Delhi, India, in 2012. In 2019 he obtained his $\mathrm{PhD}$ degree (cum laude) from University of Twente, Enschede, The Netherlands where he is currently an Assistant Professor at the Integrated Circuit Design group. From 2008 to 2010, he worked as Scientist at the Indian Space Research Organization. He also worked as Senior Design Engineer at Cadence Design Systems, India from 2012 to 2014 in the domain of high-speed serial links. His current research interests include ADCs, low-voltage low-energy circuit design, equalizers, clock and data recovery circuits. He is a recipient of the University Gold Medal in B.Tech. degree and was awarded the graduate scholarship during his Master's degree from Cadence. He serves as a reviewer for the IEEE Journal of Solid-State Circuits 